\title{
Fundamentals, progress, and future directions of nitride-based semiconductors and their composites in two-dimensional limit: A first-principles perspective to recent synthesis
}

\author{
D. Kecik, ${ }^{1, a)}$ A. Onen, ${ }^{1}$ M. Konuk, ${ }^{1}$ E. Gürbüz, ${ }^{2}$ F. Ersan, ${ }^{3}$ S. Cahangirov, ${ }^{1}$ E. Aktürk, ${ }^{3,4}$ \\ E. Durgun, ${ }^{1, b)}$ and S. Ciraci ${ }^{2, c)}$ \\ ${ }^{1}$ UNAM - National Nanotechnology Research Center and Institute of Materials Science and Nanotechnology, \\ Bilkent University, Ankara 06800, Turkey \\ ${ }^{2}$ Department of Physics, Bilkent University, Ankara 06800, Turkey \\ ${ }^{3}$ Department of Physics, Adnan Menderes University, Aydin 09100, Turkey \\ ${ }^{4}$ Nanotechnology Application and Research Center, Adnan Menderes University, Aydin 09010, Turkey
}

(Received 14 June 2017; accepted 22 November 2017; published online 22 February 2018)

\begin{abstract}
Potential applications of bulk GaN and AlN crystals have made possible single and multilayer allotropes of these III-V compounds to be a focus of interest recently. As of 2005, the theoretical studies have predicted that GaN and AIN can form two-dimensional (2D) stable, single-layer (SL) structures being wide band gap semiconductors and showing electronic and optical properties different from those of their bulk parents. Research on these 2D structures have gained importance with recent experimental studies achieving the growth of ultrathin $2 \mathrm{D}$ GaN and AlN on substrates. It is expected that these two materials will open an active field of research like graphene, silicene, and transition metal dichalcogenides. This topical review aims at the evaluation of previous experimental and theoretical works until 2018 in order to provide input for further research attempts in this field. To this end, starting from three-dimensional (3D) GaN and AlN crystals, we review 2D SL and multilayer (ML) structures, which were predicted to be stable in free-standing states. These are planar hexagonal (or honeycomb), tetragonal, and square-octagon structures. First, we discuss earlier results on dynamical and thermal stability of these SL structures, as well as the predicted mechanical properties. Next, their electronic and optical properties with and without the effect of strain are reviewed and compared with those of the 3D parent crystals. The formation of multilayers, hence prediction of new periodic layered structures and also tuning their physical properties with the number of layers are other critical subjects that have been actively studied and discussed here. In particular, an extensive analysis pertaining to the nature of perpendicular interlayer bonds causing planar GaN and AlN to buckle is presented. In view of the fact that SL GaN and AlN can be fabricated only on a substrate, the question of how the properties of free-standing, SL structures are affected if they are grown on a substrate is addressed. We also examine recent works treating the composite structures of $\mathrm{GaN}$ and AlN joined commensurately along their zigzag and armchair edges and forming heterostructures, $\delta$-doping, single, and multiple quantum wells, as well as core/ shell structures. Finally, outlooks and possible new research directions are briefly discussed. Published by AIP Publishing. https://doi.org/10.1063/1.4990377
\end{abstract}

TABLE OF CONTENTS

I. INTRODUCTION

II. 3D GaN AND AIN STRUCTURES ..........

III. 2D SL GaN AND AIN STRUCTURES ........

A. Structural optimizations, stability analyses,

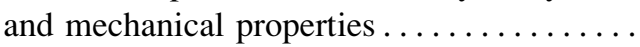

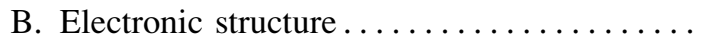

C. Effect of strain................

\footnotetext{
${ }^{a)}$ Electronic mail: kecik@unam.bilkent.edu.tr

b)Electronic mail: durgun@unam.bilkent.edu.tr

${ }^{c)}$ Electronic mail: ciraci@fen.bilkent.edu.tr
}

D. Effect of substrate............... 10 IV. MULTILAYERS OF SL-GaN AND SL-AIN. ... 11

A. Optical properties of GaN and AlN ....... 13 V. COMPOSITE STRUCTURES OF GaN/AIN.... . 14 VI. DISCUSSIONS AND CONCLUSIONS ....... 14

\section{INTRODUCTION}

Following the invention of transistor in $1948,{ }^{1,2}$ the role of micro/solid state electronics in our life has grown progressively. The success of electronics has made $\mathrm{Si}, \mathrm{Ge}$, and group III-V compound semiconductors prime materials of research of the last century. Despite the tremendous advances in silicon 
technology, specific demands in electronics and optoelectronics have brought compound semiconductors into focus. GaAs crystal with high mobility has been a hope for high speed electronics in the 1980s. Later, due to their outstanding electronic and optical properties, three-dimensional (3D) group III-V compound nitride semiconductors, namely, GaN, AlN, InN, and their heterostructures have gained importance as wide-band gap semiconductors with critical and wide range of technological applications in microwave communication, lasers, detectors, and light emitting diodes (LEDs) due to their band gap tunable in the blue-green region of the visible spectrum and ultraviolet (UV) range. ${ }^{3-9}$ Intensive research on $3 \mathrm{D}$ GaN crystals is still continuing to reveal exceptional properties and novel applications.

A new field in electronics, namely, 2D nanoelectronics has emerged subsequent to the synthesis of single-layer (SL) graphene ${ }^{10}$ in 2004. Graphene and its contenders, h-BN and $\mathrm{h}-\mathrm{MoS}_{2}$, and other transition metal dichalcogenides (TMDs) are considered potential materials for this emerging field. ${ }^{1-16}$ As early as $2005,{ }^{17}$ the possibility that Si and IIIV compounds like GaAs, GaN, and AlN can form SL honeycomb structures like graphene, was predicted by $a b$ initio calculations. Later, the stability of SL honeycomb structures of group IV elements, $\mathrm{Si}, \mathrm{Ge}, \mathrm{SiC}$, and several group III-V compounds including GaN and AlN was demonstrated based on rigorous calculations. ${ }^{18-20}$ Since the first time prediction of 2D SL graphitic (or honeycomb) structures of GaN and $\mathrm{AlN},{ }^{17}$ which will be denoted as h-GaN and h-AlN, theoretical studies continued to unveil the diverse aspects of these materials. $^{21-37}$

While several 2D SL structures, which were first predicted through first-principles calculations, were already synthesized in the laboratories, it took, however, a long time for the realization of ultrathin layers of GaN and AlN. Earlier, followed by the synthesis of single-walled nanotubes and thick-wall tubular forms of $\mathrm{GaN}$ and $\mathrm{AlN},{ }^{38-40}$ ultrathin $\mathrm{GaN}$ and AlN layer(s), or nanosheets, as well as their composites' several forms were grown on specific substrates such as sapphire, $\mathrm{Si}(111)$, 6H-SiC(0001), InGaN, single-walled carbon nanotube/graphene, etc. ${ }^{41-46}$ Regarding the synthesis of the aforementioned h-AlN, Tsipas et al. ${ }^{47}$ have demonstrated the epitaxial growth of ultrathin hexagonal form of AlN on single crystal $\operatorname{Ag}(111)$ in 2013. They prepared AlN films by plasma assisted molecular beam epitaxy on $\operatorname{Ag}(111)$ substrate of single crystals [see Fig. 1(a)]. The Ag substrate was initially cleaned by Ar ion sputtering and annealed in cycles in order to obtain flat surfaces free of $\mathrm{C}$ and $\mathrm{O}$. Al metal source and atomic nitrogen were used to epitaxially grow graphite-like AlN on the $\mathrm{Ag}(111)$ substrate. After characterization by RHEED, flat epitaxial growth of h-AlN on $\operatorname{Ag}(111)$ surface with (1x1) reconstruction was detected. There is a significant lattice mismatch of $8 \%$ between the underlying Ag substrate and layered AlN. The ultrathin AIN layers were found to have a lattice constant of $3.13 \AA$. STM image showed that AIN displays well-defined 2D islands with honeycomb symmetry on Ag substrate. ${ }^{47}$ Ultrathin AIN consisting of 12 monolayers of AlN grown on Ag substrate revealed a smaller band gap compared to the few-layer AIN/ $\mathrm{Si}(111)$ case $^{47}$ in compliance with the previous calculations for h-AlN. ${ }^{32}$ In a more recent study, few monolayers thick flat h-AlN was formed on $\mathrm{Si}(111)$ surface by molecular beam epitaxy, with lattice constant of h-AIN being $3.08 \AA .{ }^{48}$

Much recently, 2D GaN has been synthesized via graphene encapsulation, which is a significant achievement pointing GaN to be an important nanomaterial in 2D flexible optoelectronics. ${ }^{49}$ In this study, the growth of wurtzite phase of $\mathrm{GaN}$ (wz-GaN) on $\mathrm{SiC}(0001)$ was initially realized in the forms of 3D islands [see Fig. 1(b)]. Subsequent to the passivation of the high surface energy states, Frank-van der Merwe growth was promoted, allowing for $2 \mathrm{D} \mathrm{GaN}$ to be obtained. The overall process in a nutshell is that the buckled form of $2 \mathrm{D} \mathrm{GaN}$ is sandwiched between bilayer graphene top coating
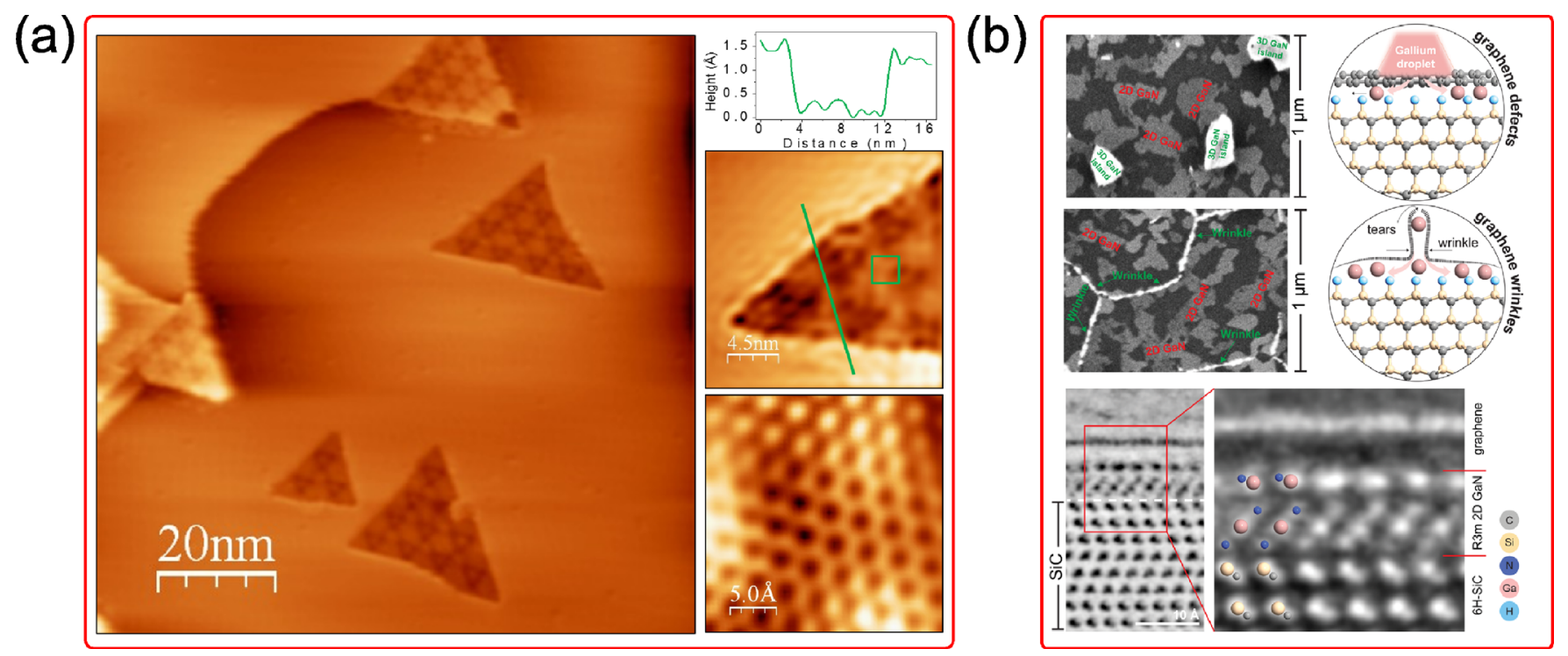

FIG. 1. (a) Overall and close-up STM views of epitaxial 2D triangular ultrathin h-AlN islands grown on $\mathrm{Ag}(111)$ substrates. Reproduced with permission from Appl. Phys. Lett. 103, 251605 (2013). Copyright 2013 AIP Publishing LLC. (b) (Top) SEM images of 2D GaN patchwork near 3D GaN islands, (bottom) STEM images and atomic models of $\mathrm{Ga}, \mathrm{N}, \mathrm{Si}$, and $\mathrm{C}$ atomic columns within graphene/2D GaN/6H-SiC(0001) heterostructure. Reproduced with permission from Al Balushi et al., Nature Mater. 15, 1166 (2016). Copyright 2016 Nature Publishing Group. 
and the underlying $6 \mathrm{H}-\mathrm{SiC}$ substrate, where hydrogenation is applied in order to passivate the dangling bonds between graphene and $\mathrm{SiC}(0001)+\mathrm{C}$ layer. The band gap of the overall structure which is achieved by electron energy loss spectroscopy (EELS) and calculated by first-principles methods using hybrid functionals (HSE), respectively, are 5.53 and $4.89 \mathrm{eV}$, significantly higher than the $3.42 \mathrm{eV}$ HSE band gap of pristine h-GaN. ${ }^{34}$ In contrast to the free-standing SL GaN and bilayer (BL) GaN forming planar layers, ultrathin 2D $\mathrm{GaN}$ grown on $\mathrm{SiC}$ is buckled owing to the $\mathrm{N}-\mathrm{Si}$ bonds with substrate.

This paper reviews several studies on SL and few-layer GaN and AlN, starting from 2005 until 2018 with an emphasis placed on their physical properties for potential technological applications. Since the theoretical studies outnumber the experimental ones within the 2D framework, the focus throughout this review work is mostly on the former. Our objective is to draw attention to seminal works with the hope of revealing new directions for future research. To this end, we present a brief outline on the physical properties of 3D $\mathrm{GaN}$ and AlN in wurtzite (wz), zincblende (zb), and cubic rocksalt (rs) structures. Then, we examine earlier works on 2D SL freestanding structures predicted for GaN and AlN in terms of their energetics, dynamical and thermal stabilities, mechanical properties, and demonstrate that they are stable and robust above room temperature. This is a significant premise that SL and few-layer GaN and AlN grown on substrates will remain stable for various applications. Understanding the electronic energy bands of the optimized structures and their optical properties is essential in order to unveil the potential of these nanomaterials in diverse optoelectronic applications. In this respect, we present an extensive discussion of the electronic and optical properties of 2D SL GaN and AlN structures. Formations of bilayers, trilayers (TL), and multilayers (ML), even layered allotropes of $\mathrm{GaN}$ and AlN like graphite and tuning their physical properties with the number of layers are among the current interests. The nature of the attractive interaction that is responsible for the binding of layers; in particular, perpendicular bonds between the layers leading to the buckling of otherwise planar GaN and AlN layers, are reviewed in detail. Whether the properties of free-standing SL GaN and AlN can be maintained when they are grown on specific substrates is another crucial feature revealed from earlier theoretical and experimental studies. Finally, we provide an analysis emphasizing the significance of the composite structures of SL GaN and AlN in fabricating novel nanomaterials forming heterostructures to construct single and multiple quantum wells, and patterned quantum dots. Interesting analyses based on the existing studies discussed in this review are partially complemented and supported by new data for providing a comparative presentation. Complementary new data are partially incorporated in Figs. 2 and 4-7, where the reader may refer for the computational details to Ref. 34 .

\section{3D GaN AND AIN STRUCTURES}

Group III-V 3D nitride semiconductors have possessed an important place in the device industry, due to their critical uses in microwave communications, LEDs, lasers, photodetectors, and transistors. wz-GaN corresponds to the thermodynamically stable phase corresponding to the global minimum. This phase has high thermal, mechanical, and chemical stability according to the experimental studies and exhibits outstanding optoelectronic properties with a wide direct band gap of 3.4-3.5 eV. ${ }^{50-58}$ The advanced epitaxial growth techniques and efficient use of $\mathrm{GaN}$ in shortwavelength blue and ultraviolet LEDs, high-power, and high-frequency devices ${ }^{6,59}$ have led to a great potential for its optoelectronic applications, corroborated with the Nobel prize for physics awarded in 2014 for the use of GaN in efficient blue LEDs. ${ }^{9}$ AlN also crystallizes in hexagonal wurtzite structure (wz-AlN) under ambient conditions. High melting point, thermal conductivity, and large bulk modulus of both 3D bulk GaN and AlN are related with their strong ionic + covalent bonds in tetrahedral directions.

$\mathrm{GaN}$ in cubic zincblende (zb-GaN) structure, on the other hand, has a slightly lower cohesive energy relative to wz-GaN. It is epitaxially grown as thin films on (011) planes of the cubic substrates. ${ }^{6}$ AlN is also stable in its bulk zincblende (zb-AlN) form, yet only in samples thinner than $2.0 \mathrm{~nm}$, where a phase transition may also occur into the rocksalt form (rs-AlN), which was observed experimentally at high pressures. ${ }^{60-62}$ High electrical resistivity ${ }^{63,64}$ and high thermal conductivity ${ }^{65}$ of AlN, as well as the wZ phase's wide and direct band-gap $\left(E_{g-d}\right)$ of nearly $4.2-4.3 \mathrm{eV}^{66-68}$ comprise remarkable features. ${ }^{59,69-74}$

The optimized atomic configurations and electronic energy bands of 3D GaN in the wz and zb structures ${ }^{34}$ are presented in Fig. 2(a), reproduced from Ref. 34. Both 3D phases are direct band gap semiconductors, with PBE$E_{g-d}=1.71 \mathrm{eV}$ and $1.55 \mathrm{eV}$, respectively, which are underestimated by $1.70-1.75 \mathrm{eV}$ with respect to the reported experimental values. ${ }^{50-58,75-77}$ Fig. 2(b) shows the optimized atomic configurations and calculated energy bands of 3D AlN in wz, zb, and rs structures for the sake of comparison. The direct band gaps of 3D wz-AlN, zb-AlN, and rs-AlN are 4.2, 3.3, and 4.6 eV, respectively. While the PBE band gaps of 3D phases of GaN and AlN are underestimated, these gaps approach the experimental values upon correction by HSE and $G_{0} W_{0}$. In Figs. 2(a) and 2(b), the energy bands corrected by HSE are also presented. Furthermore, lattice constants, Ga-N bond length, cohesive energy per Ga-N pair, bulk modulus, and some other features of 3D phases of $\mathrm{GaN}$ are listed in Table I, based on the results from Ref. 34 and several previous theoretical and experimental studies. Likewise, structural parameters, mechanical, and electronic properties of the three different 3D AlN phases are also tabulated in Table II, presented along with comparisons among earlier theoretical and experimental studies.

The cohesive energies [per $\mathrm{Ga}(\mathrm{Al})-\mathrm{N}$ pair] of $3 \mathrm{D}$ and 2D allotropes of GaN (AlN) relative to their constituent free atoms are calculated from the expression, $E_{c}$ $=E_{T}[\mathrm{Ga}(\mathrm{Al})]+E_{T}[\mathrm{~N}]-E_{T}[\mathrm{GaN}(\mathrm{AlN})]$ in terms of the total energies $E_{T}[\mathrm{Ga}(\mathrm{Al})]$ and $E_{T}[\mathrm{~N}]$ of free $\mathrm{Ga}(\mathrm{Al})$ and $\mathrm{N}$ atoms, respectively, and the optimized total energy, $E_{T}[\mathrm{GaN}(\mathrm{AlN})]$ of a specific allotrope. By subtracting the cohesive energy of the structure in the global minimum of the given III-V 
(a)

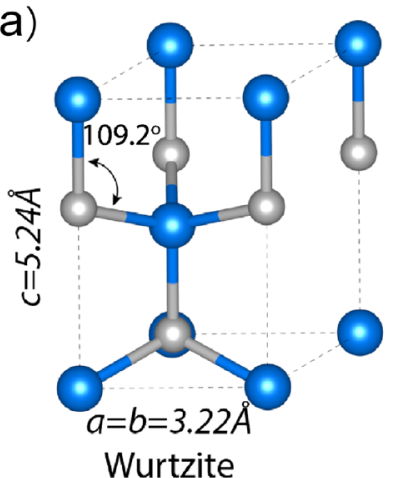

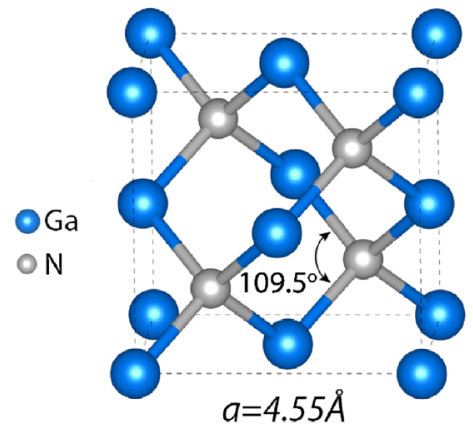

Cubic Zincblende
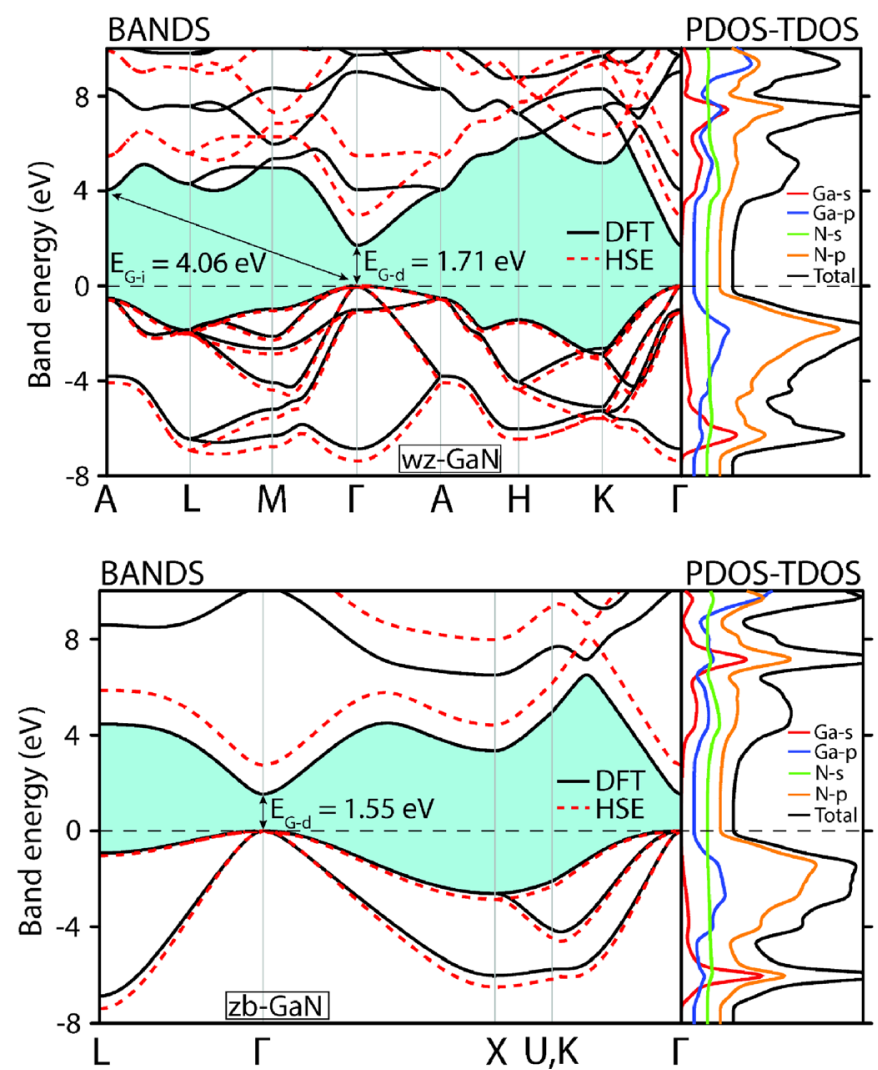
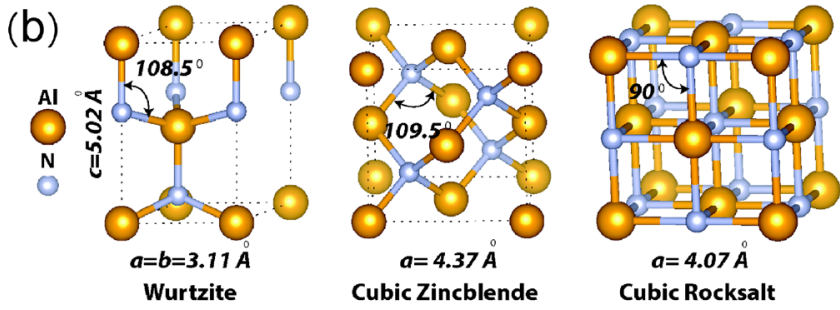

Cubic Rocksalt

PDOS - TDOS
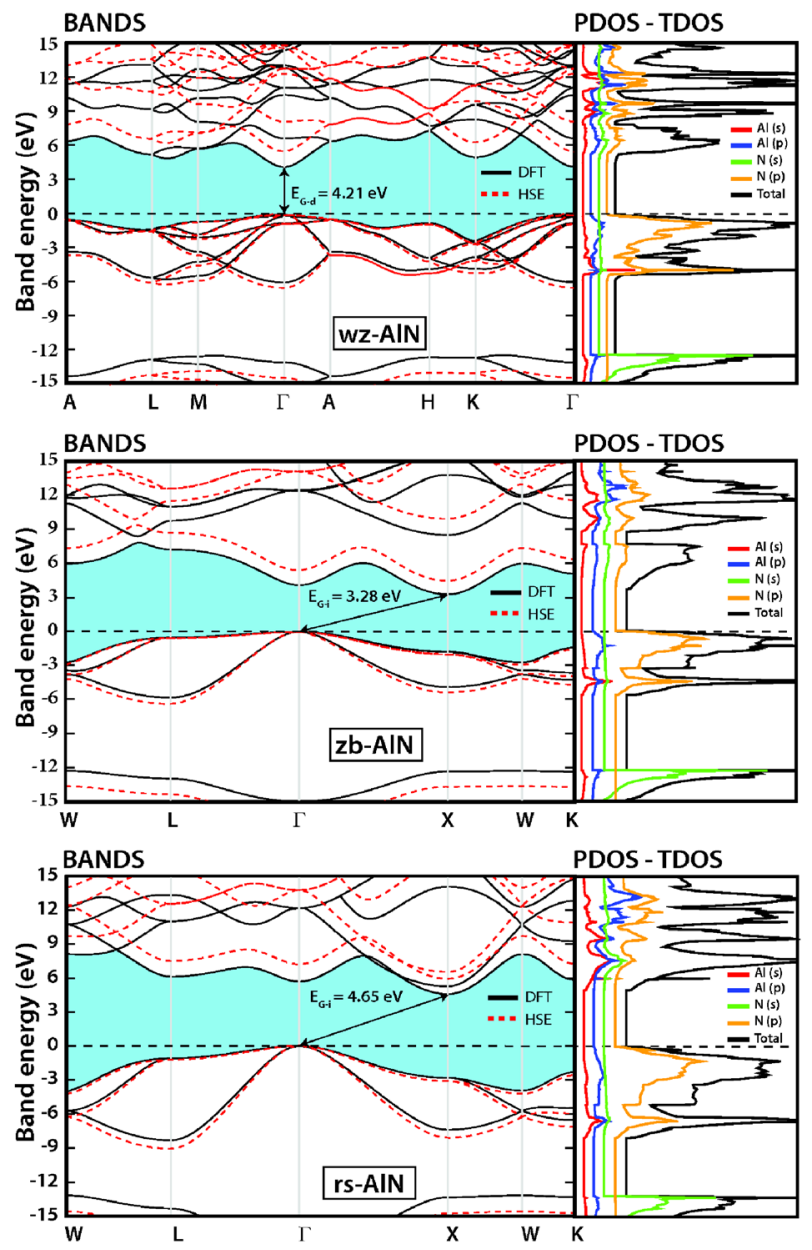

FIG. 2. (a) Top: Hexagonal and cubic conventional cells of bulk wz-GaN and zb-GaN, respectively, with lattice constants and bond angles indicated. Larger (blue) and smaller (gray) balls stand for Ga and N atoms. Middle: Electronic band structure and the total (TDOS) and partial (PDOS) densities of states of wzGaN calculated by PBE. The bands corrected by HSE are shown by the dashed red lines. The fundamental band gap of PBE + SOC calculations are shaded. The zero of energy is taken at the top of the valence band at the center of the Brillouin zone. Bottom: Same for zb-GaN. Part (a) of the figure is reproduced with permission from Onen et al., Phys. Rev. B 93, 085431 (2016). Copyright 2016 American Physical Society. (b) Top panel: Unit cell, atomic positions, and lattice parameters of the three phases of bulk AlN. Lower panels: Electronic energy band structures of wz-AlN, zb-AlN, and rs-AlN calculated by PBE + SOC, respectively. The bands corrected by HSE are shown by the dashed red lines. Part (b) of this figure is based on new data provided for the sake of comparison and complementing the existing results.

compound from those of other 3D and 2D allotropes, one obtains the formation energy $\left(E_{f}\right)$ at $\mathrm{T}=0 \mathrm{~K}$ relative to the global minimum.

\section{2D SL GaN AND AIN STRUCTURES}

Earlier theoretical studies have predicted so far two different stable structures of 2D, free-standing SL GaN, namely, planar honeycomb structure with hexagonal lattice, ${ }^{17,20,34,107} \mathrm{~h}-\mathrm{GaN}$, and square-octagon ${ }^{108,109}$ (so-GaN) with square lattice. Much recently, porous hexagonal and tetragonal forms of SL GaN, with larger surface areas than that of h-GaN, namely, $\mathrm{H}-\mathrm{GaN}$ and T-GaN were found to be stable via phonon calculations. ${ }^{110}$ Stable 2D SL structures of AlN are planar h-AlN, ${ }^{17,20,107}$ tetragonal t-AlN, ${ }^{24}$ and soAlN. ${ }^{108,109}$ In this paper, only the $\mathrm{h}-, \mathrm{t}-$ and so-structures will be considered.

Following the prediction of SL h-GaN, Freeman $e t a l .{ }^{21}$ investigated by first-principles calculations the ultrathin films of AlN and GaN and demonstrated that the [0001] surfaces adopt a graphite-like structure. The electronic properties of hydrogenated III-V sheets from first-principles were investigated by Wang et al., ${ }^{22}$ who have found that the hydrogenated monolayer $\mathrm{GaN}$ and $\mathrm{AlN}$ are wide-gap semiconductors. $\mathrm{GaN}$ 
TABLE I. 3D wurtzite and zincblende GaN: Lattice constants $a=b$ and $c$; Ga-N bond length $d$, cohesive energy $E_{c}$ per Ga-N pair; bulk modulus $B$, Poisson's ratio $\nu$, charge transfer $Q_{b}^{*}$ from cation to anion through Bader analysis, ${ }^{78}$ Born effective charges $Z^{*}$, and direct band gap $E_{g-d}$ of wz- and zb-GaN crystals calculated by using PBE, HSE (with different mixing parameters $\alpha$ ), and $G_{0} W_{0}$ approaches. For the sake of comparison, values obtained from the previous theoretical studies and experiments are also included.

\begin{tabular}{|c|c|c|c|c|c|c|c|c|c|c|}
\hline & & $\begin{array}{c}a \\
(\AA)\end{array}$ & $\begin{array}{c}c \\
(\AA)\end{array}$ & $\begin{array}{c}d \\
(\AA)\end{array}$ & $\begin{array}{c}E_{c} \\
(\mathrm{eV} / \mathrm{GaN})\end{array}$ & $\begin{array}{c}B \\
(\mathrm{GPa})\end{array}$ & $\begin{array}{c}\nu \\
(\%)\end{array}$ & $\begin{array}{l}Q_{b}^{*} \\
\text { (e) }\end{array}$ & $\begin{array}{l}Z^{*} \\
\text { (e) }\end{array}$ & $\begin{array}{l}E_{g-d} \\
(\mathrm{eV})\end{array}$ \\
\hline 3D-wz & PBE & 3.22 & 5.24 & 1.97 & 8.76 & 171 & 0.18 & 1.54 & 2.63 & 1.71 \\
\hline 3D-wz & $\operatorname{HSE}(\alpha=0.25 / 0.35)$ & - & - & - & - & - & - & - & - & $2.96 / 3.48$ \\
\hline $3 \mathrm{D}-\mathrm{wz}$ & $G_{0} W_{0}$ & - & - & - & - & - & - & - & - & 3.03 \\
\hline $3 \mathrm{D}-\mathrm{wz}$ & LDA/GGA ${ }^{79}$ & $3.16 / 3.22$ & $5.15 / 5.24$ & - & - & - & - & - & - & $2.12 / 1.74$ \\
\hline $3 D-w z$ & $\mathrm{LDA} / \mathrm{GGA}^{80}$ & $3.16 / 3.22$ & $5.14 / 5.24$ & - & - & $197.4 / 172.2$ & - & - & - & $G_{0} W_{0}: 3.37$ \\
\hline 3D-wz & $\mathrm{PBE}^{81}$ & 3.14 & - & - & - & 215 & - & - & 2.64 & - \\
\hline 3D-wz & $\mathrm{PBE}^{82}$ & 3.20 & - & - & - & - & - & - & 2.72 & - \\
\hline $3 \mathrm{D}-\mathrm{wz}$ & $\mathrm{PBE}^{83}$ & 3.15 & - & - & - & 195 & - & - & - & - \\
\hline 3D-wz & $\mathrm{PBE}^{50}$ & 3.19 & - & - & - & - & - & - & - & 1.83 \\
\hline $3 \mathrm{D}-\mathrm{wz}$ & $\mathrm{PBE} / \mathrm{HSE}^{84}$ & $3.15 / 3.18$ & $5.14 / 5.17$ & - & - & - & - & - & $2.58 / 2.64$ & - \\
\hline 3D-wz & $\mathrm{PBE}^{85}$ & 3.17 & 5.15 & - & - & 207 & - & - & - & 2.22 \\
\hline 3D-wz & $\mathrm{HSE}^{86}$ & 3.20 & 5.20 & - & - & - & - & - & - & 3.21 \\
\hline $3 \mathrm{D}-w \mathrm{z}$ & $\operatorname{HSE}(\alpha=0.25 / 0.30)^{79}$ & $3.18 / 3.17$ & $5.17 / 5.16$ & - & - & - & - & - & - & $3.27 / 3.48$ \\
\hline 3D-wz & $\mathrm{LDA} / \mathrm{QS} G W^{87}$ & $3.16 /-$ & $5.14 /-$ & - & - & - & - & - & - & $-/ 3.60$ \\
\hline 3D-wz & $G_{0} W_{0}^{88}$ & 3.19 & 5.19 & - & - & - & - & - & - & 3.24 \\
\hline 3D-wz & Expt. $^{50-58}$ & 3.19 & 5.19 & - & 9.06 & $188,195,205,237,245$ & 0.20 & - & 2.65 & $3.40-3.50$ \\
\hline $3 \mathrm{D}-\mathrm{zb}$ & $\mathrm{PBE}$ & 4.55 & - & 1.97 & 8.75 & 170 & 0.34 & 1.52 & 2.68 & 1.55 \\
\hline $3 \mathrm{D}-\mathrm{zb}$ & $\mathrm{LDA} / \mathrm{GGA}^{80}$ & $4.46 / 4.55$ & - & - & - & $188.8 / 172.0$ & - & - & - & $\begin{array}{c}-/ 1.57 \\
\left.\text { (HSE:2.59, } G_{0} W_{0}: 3.16\right)\end{array}$ \\
\hline $3 \mathrm{D}-\mathrm{zb}$ & $\operatorname{HSE}(\alpha=0.25 / 0.35)$ & - & - & - & - & - & - & - & - & $2.74 / 3.30$ \\
\hline $3 \mathrm{D}-\mathrm{zb}$ & $G_{0} W_{0}$ & - & - & - & - & - & - & - & - & 2.85 \\
\hline $3 \mathrm{D}-\mathrm{zb}$ & $\mathrm{PBE}^{83}$ & 4.46 & - & - & - & 183 & - & - & - & - \\
\hline $3 \mathrm{D}-\mathrm{zb}$ & $\mathrm{PBE}^{81}$ & 4.45 & - & - & - & 207 & - & - & 2.65 & - \\
\hline $3 \mathrm{D}-\mathrm{zb}$ & $\mathrm{PBE}^{89}$ & 4.56 & - & - & - & - & - & - & - & 1.66 \\
\hline $3 \mathrm{D}-\mathrm{zb}$ & $G_{0} W_{0}^{90-92}$ & 4.5 & - & - & - & - & - & - & - & $2.79,2.88,3.09$ \\
\hline $3 \mathrm{D}-\mathrm{zb}$ & Expt. $^{52,75-77}$ & $4.54,4.50$ & - & - & 8.90 & $185-190$ & 0.37 & - & - & 3.30 \\
\hline
\end{tabular}

and AlN half-decorated by $\mathrm{H}$ or $\mathrm{F}$ were also shown to display magnetic properties. ${ }^{23}$ Singh et $a .^{24}$ examined the growth, stability, and electronic structures of SL h-GaN, h-AlN, and tAIN theoretically in the presence of underlying substrates. They proposed that metal diborides enable epitaxial growth as a result of large GaN adsorption energies and ionic bonding, while TMDs, which are poor stabilizers for GaN, serve as promising constituents to form GaN/TMD heterostructures. A vertically stacked $\mathrm{AlN} / \mathrm{MoS}_{2}$ heterostructure was modeled and proposed recently, where the authors examined the effects of interfacial defects and found significant interaction between both structures and tuned the band alignment by inducing vacancies and by doping. ${ }^{111}$

The effect of compressive strain on h-GaN was shown by Gao et al., ${ }^{27}$ which induced buckling of h-GaN, revealing a direct band gap, unlike the planar conformation of SL h$\mathrm{GaN}$. The external electric field applied normal to the h-GaN sheet also leads to a transformation from planar to buckled structure. h-GaN maintains the buckled structure once $\mathrm{Ga}$ and $\mathrm{N}$ atoms are hydrogenated. It has been predicted via structural optimization and phonon calculations that the planar graphitic structure of few-layer h-GaN layers is not the energetically most favorable phase; but it reconstructs into the so-called haeckelite structure with covalent interlayer bonds forming alternating octagonal and square, i.e., sorings. ${ }^{28}$ A prospective realization of few-layer of haeckelite
GaN (so-GaN), predicted to have a direct band gap is tempting for its future optoelectronic applications.

Following the comprehensive stability analysis and electronic structure study of SL group III-V compounds, ${ }^{17,20}$ further theoretical studies on ultrathin AIN indicate that h-AIN is a lower energy configuration only for small thicknesses. ${ }^{21}$ However, upon applying epitaxial strain, stable hexagonal structures can still be obtained for larger thicknesses. ${ }^{29}$ Firstprinciples calculations so far have shown that SL h-GaN and $\mathrm{h}$-AlN constituted from the same $\mathrm{N}$ ion and isovalent cations $\mathrm{Ga}$ and $\mathrm{Al}$, exhibit similar properties; in similar structures, they are almost lattice matched. In fact, in-plane, commensurate, composite structures of $(\mathrm{GaN})_{n} /(\mathrm{AlN})_{m}$ were predicted to form SL semiconductors with tunable properties, such as single and multiple quantum wells, 2D patterned quantum dots, and $\delta$-doping. ${ }^{35}$

\section{A. Structural optimizations, stability analyses, and mechanical properties}

h-GaN and h-AlN, which were predicted earlier to have planar honeycomb structures, ${ }^{17,20}$ were demonstrated to possess dynamical stability for the optimized lattice constants of $3.09 \AA$ and $3.20 \AA$, respectively, calculated using LDA. ${ }^{20}$ The optimized atomic structure of free standing SL h-GaN, together with its primitive cell, lattice constants, and charge 
TABLE II. 3D wurtzite, zincblende, and rocksalt AlN: Lattice constants $a=b$ and $c$; Al-N bond length $d$, cohesive energy $E_{c}$ per Al-N pair; bulk modulus $B$, Poisson's ratio $\nu$, charge transfer $Q_{b}^{*}$ from cation to anion through Bader analysis, ${ }^{78}$ Born effective charges $Z^{*}$, and $E_{g-d / i}(\mathrm{~d}=$ direct, i $=$ indirect) of wz-, zb-, and rs-AlN crystals calculated by using PBE, HSE (with different mixing parameters $\alpha$ ), and $G_{0} W_{0}$ approaches. For the sake of comparison, values obtained from the previous theoretical studies and experiments are also included.

\begin{tabular}{|c|c|c|c|c|c|c|c|c|c|c|}
\hline & & $\begin{array}{c}a \\
(\AA)\end{array}$ & $\begin{array}{c}c \\
(\AA)\end{array}$ & $\begin{array}{c}d \\
(\AA)\end{array}$ & $\begin{array}{c}E_{c} \\
(\mathrm{eV} / \mathrm{AlN})\end{array}$ & $\begin{array}{c}B \\
(\mathrm{GPa})\end{array}$ & $\begin{array}{c}\nu \\
(\%)\end{array}$ & $\begin{array}{l}Q_{b}^{*} \\
(\mathrm{e})\end{array}$ & $\begin{array}{l}Z^{*} \\
(\mathrm{e})\end{array}$ & $\begin{array}{c}E_{g} \\
(\mathrm{eV})\end{array}$ \\
\hline 3D-wz & $\mathrm{PBE}$ & 3.11 & 5.02 & 1.90 & 12.10 & 194.1 & 0.21 & 1.38 & 2.52 & $4.21(\mathrm{~d})$ \\
\hline 3D-wz & $\operatorname{HSE}(\alpha=0.25 / 0.35)$ & - & - & - & - & - & - & - & - & $5.42 / 5.96$ \\
\hline $3 \mathrm{D}-\mathrm{wz}$ & $\mathrm{LDA} / \mathrm{QP}^{93}$ & $3.07 /-$ & - & - & - & - & - & - & - & 4.67 (d)/6.80 (d) \\
\hline 3D-wz & $\mathrm{PBE}^{66}$ & 3.11 & 4.98 & - & - & - & - & - & - & $4.22(\mathrm{~d})$ \\
\hline $3 \mathrm{D}-\mathrm{wz}$ & $\mathrm{PBE}^{94}$ & 3.06 & - & 1.87 & - & - & - & 0.37 & - & $\sim 5.00(\mathrm{~d})$ \\
\hline 3D-wz & $\mathrm{PBE}^{95}$ & 3.12 & 5.01 & - & - & 188.0 & - & - & - & - \\
\hline 3D-wz & LDA/GGA ${ }^{96}$ & $3.10 / 3.14$ & $4.97 / 5.05$ & - & - & - & - & - & - & $4.32 / 3.96(\mathrm{~d})$ \\
\hline 3D-wz & LDA/GGA ${ }^{80}$ & $3.09 / 3.13$ & $4.95 / 5.02$ & - & - & $210.8 / 187.2$ & - & - & - & $G_{0} W_{0}: 6.14$ \\
\hline $3 \mathrm{D}-\mathrm{wz}$ & $\mathrm{PBE}^{67}$ & 3.08 & 4.94 & - & - & - & - & 0.46 & - & $4.30(\mathrm{~d})$ \\
\hline 3D-wz & $\mathrm{PBE}^{32}$ & 3.11 & 5.01 & 1.90 & 12.28 & - & - & 2.35 & - & $4.20(\mathrm{~d})$ \\
\hline $3 \mathrm{D}-\mathrm{wz}$ & LDA/QSG $G W^{87}$ & $3.11 /-$ & $4.97 /-$ & - & - & - & - & - & - & $-/ 6.19$ \\
\hline 3D-wz & LDA/QSG $G W^{98}$ & - & - & - & - & - & - & - & - & $4.24 / 6.77$ \\
\hline 3D-wz & $\mathrm{MD}^{68}$ & - & - & - & 11.52 & 219.7 & $0.18-0.29$ & - & - & - \\
\hline $3 \mathrm{D}-\mathrm{wz}$ & Expt. $^{54,97,99}$ & 4.37 & - & - & 11.52 & $210,207.9$ & $0.22-0.29$ & - & - & 6.28 \\
\hline $3 \mathrm{D}-\mathrm{zb}$ & PBE & 4.37 & - & 1.90 & 12.06 & 193.5 & 0.25 & 1.22 & 2.72 & 3.29 (i) \\
\hline $3 \mathrm{D}-\mathrm{zb}$ & $\operatorname{HSE}(\alpha=0.25 / 0.35)$ & - & - & - & - & - & - & - & - & $4.50 / 4.98$ \\
\hline $3 \mathrm{D}-\mathrm{zb}$ & $\mathrm{PBE}^{100}$ & 4.38 & - & - & - & 195.5 & - & - & - & 3.31 (i) \\
\hline $3 \mathrm{D}-\mathrm{zb}$ & $\mathrm{PBE}^{94}$ & 4.30 & - & 1.86 & - & - & - & 0.33 & - & 4.00 (i) \\
\hline $3 \mathrm{D}-\mathrm{zb}$ & $\mathrm{PBE}^{101}$ & 4.30 & - & - & - & 209 & - & - & - & - \\
\hline $3 \mathrm{D}-\mathrm{zb}$ & $\mathrm{PBE}^{102}$ & 4.36 & - & - & - & 208 & - & - & - & - \\
\hline $3 \mathrm{D}-\mathrm{zb}$ & LDA/GGA ${ }^{96}$ & $4.35 / 4.41$ & - & - & - & - & - & - & - & $3.24 / 3.31(\mathrm{i})$ \\
\hline $3 \mathrm{D}-\mathrm{zb}$ & $\mathrm{LDA} / \mathrm{GGA}^{80}$ & $4.34 / 4.40$ & - & - & - & $212.0 / 193.2$ & - & - & - & $\begin{array}{c}-/ 3.31 \\
\left.\text { (HSE: } 4.32, G_{0} W_{0}: 5.16\right)\end{array}$ \\
\hline $3 \mathrm{D}-\mathrm{zb}$ & $\mathrm{PBE}^{67}$ & 4.37 & - & - & - & - & - & 0.44 & - & 3.20 (i) \\
\hline $3 \mathrm{D}-\mathrm{zb}$ & $\mathrm{LDA} / \mathrm{QS} G W^{98}$ & - & - & - & - & - & - & - & - & $3.24 / 5.64$ \\
\hline $3 \mathrm{D}-\mathrm{zb}$ & $\mathrm{MD}^{68}$ & - & - & - & 11.52 & 220.2 & - & - & - & - \\
\hline $3 \mathrm{D}-\mathrm{zb}$ & Expt. ${ }^{103,104}$ & 4.38 & - & 1.89 & - & - & - & - & - & 5.3 (i) \\
\hline 3D-rs & PBE & 4.07 & - & 2.02 & 11.72 & 255.9 & 0.16 & 1.20 & 3.02 & $4.52(\mathrm{i})$ \\
\hline 3D-rs & $\operatorname{HSE}(\alpha=0.25 / 0.35)$ & - & - & - & - & - & - & - & - & $5.90 / 6.44$ \\
\hline 3D-rs & $\mathrm{PBE}^{100}$ & 4.07 & - & - & - & 254.3 & - & - & - & 4.40 (i) \\
\hline 3D-rs & $\mathrm{PBE}^{105}$ & 4.07 & - & - & - & - & - & 1.25 & - & $4.53(\mathrm{i})$ \\
\hline 3D-rs & $\mathrm{PBE}^{94}$ & 3.98 & - & 1.99 & - & - & - & 0.25 & - & 5.00 (i) \\
\hline 3D-rs & $\mathrm{PBE}^{106}$ & 3.98 & - & - & - & 329 & - & - & - & - \\
\hline 3D-rs & LDA/GGA ${ }^{96}$ & $4.01 / 4.07$ & - & - & - & - & - & - & - & $4.67 / 4.43$ (i) \\
\hline 3D-rs & LDA/QSG $W^{98}$ & - & - & - & - & - & - & - & - & $4.48 / 7.01$ \\
\hline 3D-rs & $\mathrm{MD}^{68}$ & - & - & - & 11.0 & 226.8 & - & - & - & - \\
\hline
\end{tabular}

distribution around Ga-N bonds is shown in Fig. 3(a). Three of each Ga-s $s p^{2}$ and $\mathrm{N}-s p^{2}$ hybrid orbitals form ionic $\sigma$-bonds along Ga-N bonds arranged as a hexagon and provide the strength of h-GaN. Their $p_{z}$ orbitals perpendicular to the hGaN plane maintain the planar geometry. The isosurfaces of the total charge density represent the electron distribution over the hexagons, with $\mathrm{Ga}$ and $\mathrm{N}$ atoms alternatingly placed at the corners. Ga-N bonds in 3D wz(zb)-GaN, constructed of tetrahedrally coordinated $s p^{3}$ hybrid orbitals, appear to be $0.12 \AA$ longer than those in h-GaN, implying stronger bonds for the 2D structure of h-GaN. However, the cohesive energy of the four-fold coordinated 3D wz-GaN crystal is $0.70 \mathrm{eV}$ per bond higher than that of $\mathrm{h}-\mathrm{GaN}$, pointing to a local minimum in the Born-Oppenheimer (BO) surface for the latter. The lattice constants $a=b$, bond length $d$, cohesive energy $E_{c}$, in-plane stiffness $C$, Poisson's ratio $\nu$, charge transfer $Q_{b}^{*}$, Born effective charge values $Z^{*}$, and fundamental band gaps
$E_{g}$ calculated by different methods are presented in Table III, in comparison to the results of previous theoretical and experimental works. A recent PBE calculation for $\mathrm{h}-\mathrm{GaN}^{34}$ predicts $a$ and $d$ values (3.21 and $1.85 \AA$, respectively), supported with the previous theoretical results. ${ }^{20,26,107,112}$

Two free-standing, SL structures of 2D AlN, namely, hAlN and t-AlN, are displayed in Fig. 4(a). The first one has a structure similar to h-GaN, conformed in a hexagonal planar symmetry. The other free standing $2 \mathrm{D}$ phase, t-AIN possesses a non-planar tetragonal symmetry, with Al-N bonds coordinated in $90^{\circ}$ angle. Structural parameters, $E_{c}, C, \nu$, $Q_{b}^{*}, Z^{*}$, and $E_{g}$ values of the SL free-standing, h-, and t-AIN phases, computed by using different methods are tabulated in Table IV, presented also in comparison to few experimental studies. The calculated $a, d$, and $E_{c}$ values of h-AlN are $3.13,1.81 \AA$, and $10.56 \mathrm{eV}$, respectively, in fair agreement with the previous calculations. ${ }^{20,32,118}$ On the other hand, $a$, 


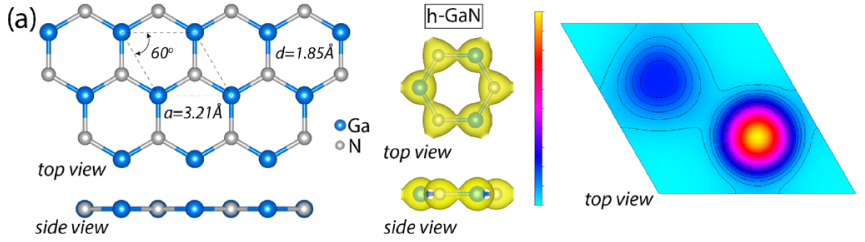

(b)

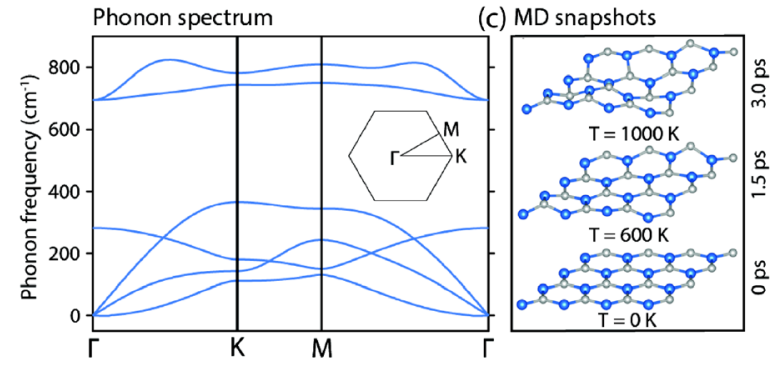

(d)

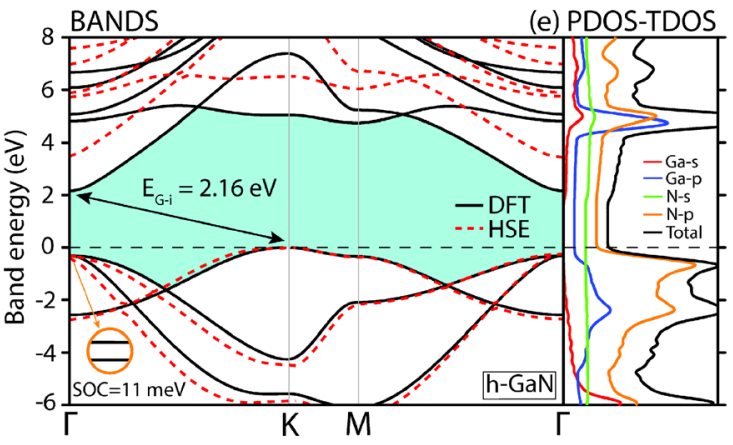

FIG. 3. (a) Top and side views of the optimized atomic structure of h-GaN, together with the isosurfaces of the total charge density of a hexagon and charge density contour plots of $\mathrm{Ga}-\mathrm{N}$ bond in a horizontal plane. (b) Calculated phonon dispersion curves. (c) Snapshots of the atomic configurations in MD simulations at 0,600, and $1000 \mathrm{~K}$ temperatures, in which honeycomb like structures are maintained. (d) Electronic energy band structure of the optimized structure of $\mathrm{h}-\mathrm{GaN}$ is presented along the symmetry directions of the Brillouin zone. Zero of energy is set to the top of the valence band. The fundamental band gap between conduction and valence bands are shaded and indirect band gap $E_{g-i}$ is indicated. The splitting of the degenerate bands at the top of the valance band at the $\Gamma$-point due to spin-orbit coupling is shown by the inset. PBE bands corrected by the HSE method are shown by the dashed lines. (e) TDOS and PDOS. Reproduced with permission from Onen et al., Phys. Rev. B 93, 085431 (2016). Copyright 2016 American Physical Society. $d$, and $E_{c}$ values of t-AlN were calculated by $\mathrm{PBE}$ as 3.63 , $1.90 \AA$, and $10.42 \mathrm{eV}$, respectively. Both $2 \mathrm{D}$ AlN phases have nearly $1.6 \mathrm{eV}$ lower cohesive energies with respect to the bulk wZ- and zb-AlN phases. While bond-length of bulk wZ- and zb-AlN are identical with that of SL t-AIN, h-AlN has a bond length value nearly $0.1 \AA$ shorter than that of $3 \mathrm{D}$ wZ-AlN, indicating stronger Al-N bonds for h-AlN. In compliance with this conclusion, the cohesive energy of h-AlN is $140 \mathrm{meV}$ higher than that of t-AlN.

Recently, the so-called haeckelite structure of freestanding SL so-GaN and so-AlN consisting of squares and octagons were demonstrated from first-principles to be stable. ${ }^{108,109}$ In these structures, one distinguishes bonds of two different lengths due to the different bond angles: $90.1^{\circ}$ and $86.2^{\circ}$. The shorter ones are between the adjacent octagons, while the slightly longer ones are between square and octagon rings as demonstrated in Fig. 5. Values of the relevant properties related with the atomic structure, cohesive energy, elastic properties, etc., calculated for so-GaN and so-AlN are presented in Tables III and IV. ${ }^{109}$

Further to the earlier LDA phonon calculations,${ }^{20}$ recent $a b$ initio phonon calculations using PBE presented in Figs. $3-5^{34,109}$ confirmed the dynamical stability of SL 2D allotropes of GaN and AlN at $\mathrm{T}=0 \mathrm{~K}$. This situation indicates that SL h-GaN, so-GaN, h-AlN, t-AlN, and so-AlN remain dynamically stable in spite of the fact that they have negative formation energies. ${ }^{32,34,109}$ Accordingly, they correspond to a local minima on the BO surface. However, thermal excitations may induce instabilities in the free-standing 2D structures, even though all phonon frequencies in the Brillouin zone come out to be positive. For instance, a structure can dissociate even at low temperatures, when the local minimum of a given phase is shallow in the BO surface. In order to assure that these minima are truly deep such that the SL structures preserve their stability under thermal excitations, earlier $a b$ initio finite temperature molecular dynamics (MD) calculations were performed in the range of $0-1000 \mathrm{~K}$, to confirm the high-temperature stabilities of all SL GaN and AlN phases. ${ }^{32,34,109}$ Accordingly, all free-standing SL

TABLE III. Free-standing, SL h-GaN, and so-GaN: Optimized lattice constant $a$; Ga-N bond length $d$ (two different Ga-N bond lengths $d_{1} / d_{2}$ for so-GaN), cohesive energy $E_{c}$ per Ga-N pair; in-plane stiffness $C$ (uniaxial strain along $x$-axis/along diagonal of $\sqrt{2} \times \sqrt{2}$ of so-GaN), Poisson's ratio $\nu$ (uniaxial strain along $x$-axis/along diagonal of $\sqrt{2} \times \sqrt{2}$ of so-GaN), charge transfer $Q_{b}^{*}$ from Ga to $\mathrm{N}$, Born effective charge $Z^{*}$, and indirect $E_{g-i}$ band gaps calculated using different methods.

\begin{tabular}{|c|c|c|c|c|c|c|c|c|c|}
\hline & & $\begin{array}{c}a \\
(\AA)\end{array}$ & $\begin{array}{c}d \\
(\AA)\end{array}$ & $\begin{array}{c}E_{c} \\
(\mathrm{eV} / \mathrm{GaN})\end{array}$ & $\begin{array}{c}C \\
(\mathrm{~N} / \mathrm{m})\end{array}$ & $\begin{array}{c}\nu \\
(\%)\end{array}$ & $\begin{array}{l}Q_{b}^{*} \\
(\mathrm{e})\end{array}$ & $\begin{array}{l}Z^{*} \\
\text { (e) }\end{array}$ & $\begin{array}{l}E_{g-i} \\
(\mathrm{eV})\end{array}$ \\
\hline $\mathrm{h}$ & $\mathrm{PBE} / \mathrm{HSE} / G_{0} W_{0}{ }^{34}$ & 3.21 & 1.85 & 8.29 & 109.8 & 0.43 & 1.50 & 3.08 & $2.16 / 3.42 / 4.55$ \\
\hline $\mathrm{h}$ & $\mathrm{LDA}^{20}$ & 3.20 & 1.85 & 12.74 & 110 & 0.48 & 1.70 & - & $2.27\left(G W_{0}: 5.0\right)$ \\
\hline $\mathrm{h}$ & $\mathrm{PBE}^{112}$ & 3.21 & 1.85 & - & 109.4 & 0.43 & - & - & - \\
\hline $\mathrm{h}$ & $\mathrm{PBE}^{107}$ & - & 1.85 & 8.38 & - & - & - & - & 2.17 \\
\hline $\mathrm{h}$ & $\mathrm{PBE}^{26}$ & - & 1.87 & 8.06 & - & - & - & - & $1.87\left(G W_{0}: 4.14\right)$ \\
\hline $\mathrm{h}$ & $G_{0} W_{0}{ }^{113}$ & 3.17 & - & - & - & - & - & - & 4.27 (LDA: 2.36 ) \\
\hline $\mathrm{h}$ & $\mathrm{PBE}^{30}$ & 3.25 & - & - & - & - & 1.34 & 3.23 & HSE: $3.23 / G_{0} W_{0}: 4.00$ \\
\hline $\mathrm{h}$ & Expt./HSE ${ }^{49}$ & $3.13 /-$ & - & - & - & - & - & - & $5.53 / 4.89$ \\
\hline $\mathrm{h}$ & $G_{0} W_{0}^{114}$ & 3.15 & - & - & - & - & - & - & 4.10 \\
\hline so & $\mathrm{PBE}^{109}$ & 6.42 & $1.91 / 1.83$ & 8.08 & $41.63 / 125$ & $0.76 / 0.29$ & - & - & 1.85 (HSE:3.37) \\
\hline so & $\mathrm{PBE}^{108}$ & - & $1.95 / 1.85$ & - & - & - & - & - & 1.60 \\
\hline so & $\mathrm{PBE} / \mathrm{HSE}^{110}$ & 6.41 & $\sim 1.83$ & - & - & - & - & - & $1.89 / 3.12$ \\
\hline
\end{tabular}


(a)

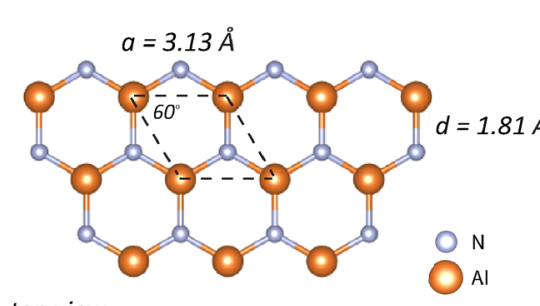

top view

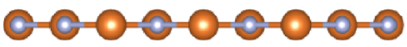

side view

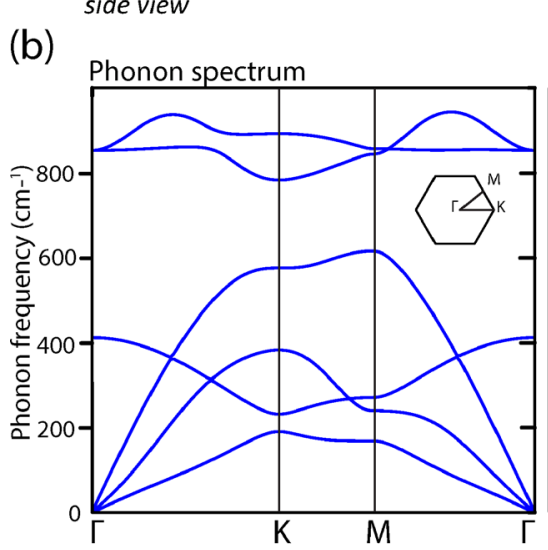

MD snapshots

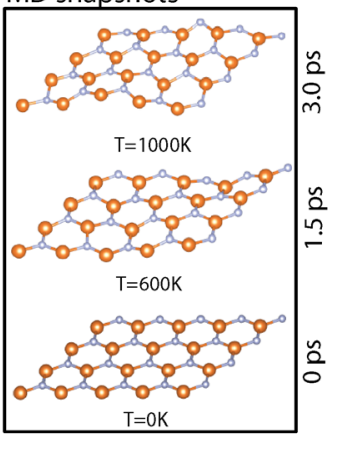

(c)

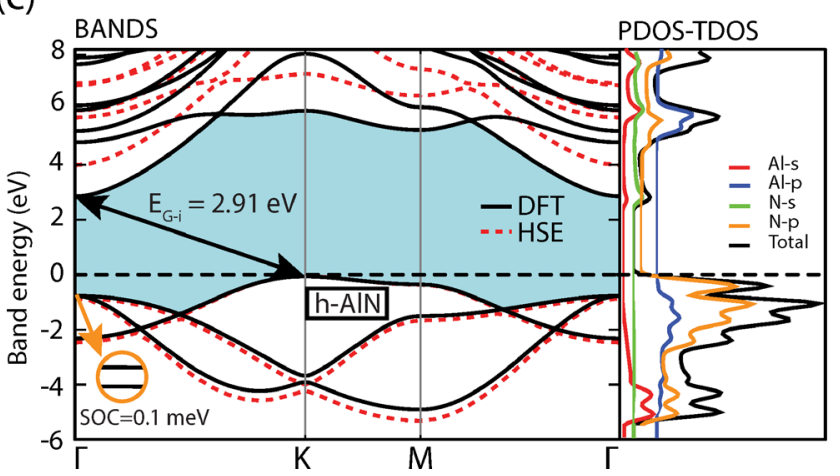

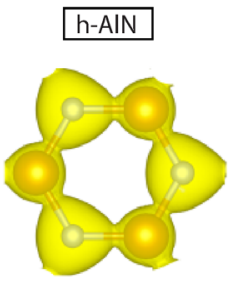

top view

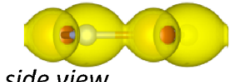

side view

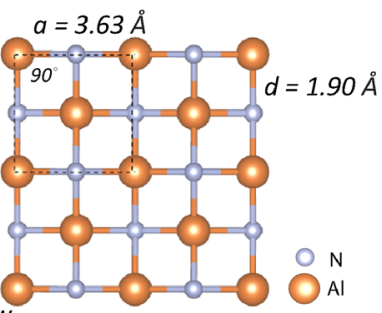

top view

side view

$0=0=0 \div 8^{2=0.56 \AA}$

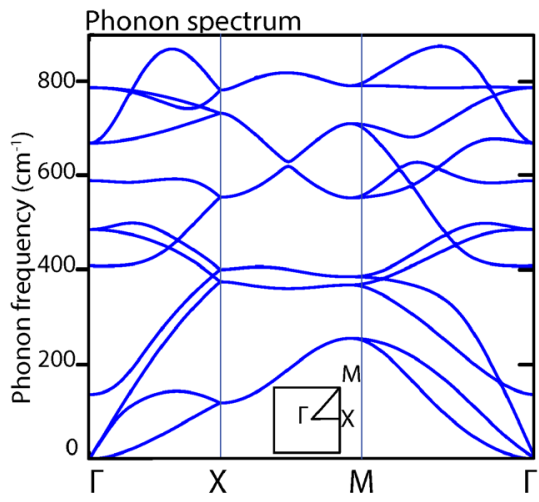

MD snapshots
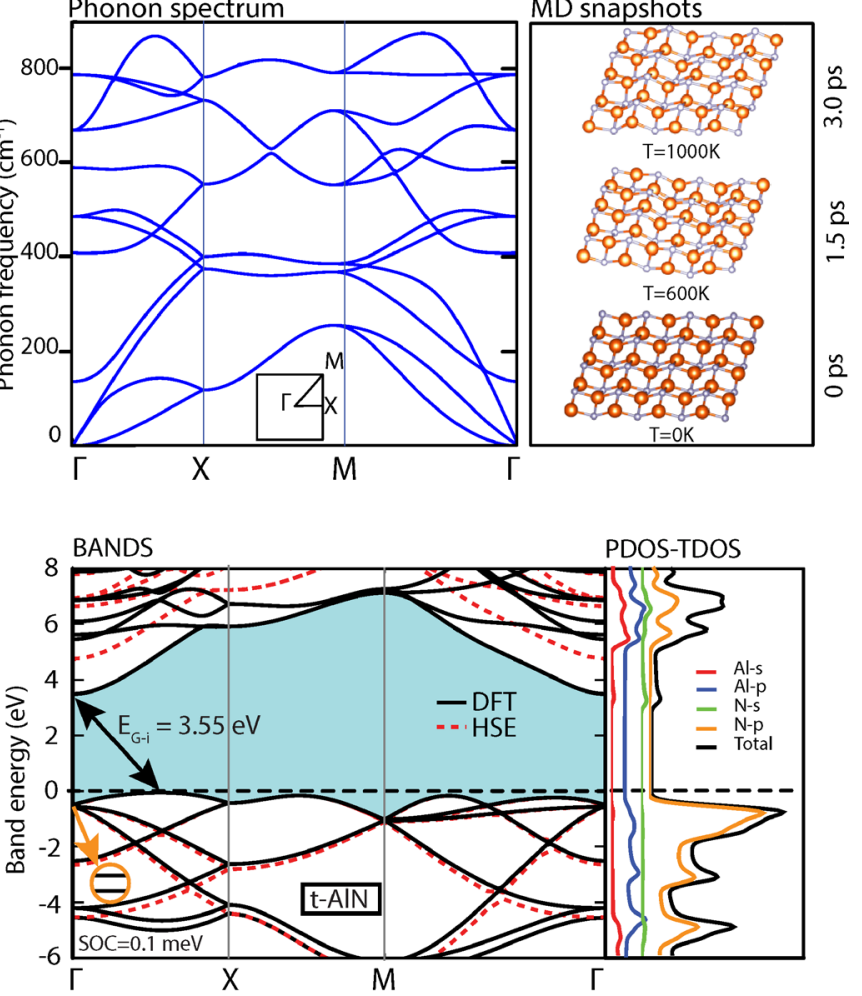

FIG. 4. (a) Top and side views of the optimized atomic structure of h-AlN and t-AlN. Primitive unit cells are delineated by dashed lines. Isosurfaces of the total charge density are also shown. (b) Phonon dispersion curves and ab initio MD snapshots of atomic configurations at $\mathrm{T}=1000,600$, and $0 \mathrm{~K}$ calculated for hAIN and t-AIN. (c) Electronic energy band structures and the corresponding TDOS and PDOS calculated by PBE and corrected by HSE (shown by dashed lines) for h-AlN and t-AIN. Fundamental band gap between the conduction and valence bands are shaded and the indirect band gap $E_{g-i}$ is indicated. This figure is based on new data provided for the sake of comparison and complementing the existing results.

structures, namely, h-GaN, so-GaN, h-AlN, t-AlN, and soAlN remained stable for 3 ps at temperatures as high as $1000 \mathrm{~K}$, assuring stability for device operations at room temperature and slightly above it. ${ }^{32,34,109}$ Concluding this section, we note that the dynamical stability tests have only limited significance and assure that the $2 \mathrm{D}$ structure at hand is stable if prepared experimentally. Moreover, the stability of SL and ML structures are enhanced if they are grown on substrates.

\section{B. Electronic structure}

In few most recent studies, ${ }^{32-34}$ the electronic and optical properties of h-GaN and h-AlN SL and ML structures were compared with those of $3 \mathrm{D} \mathrm{GaN}$ and $\mathrm{AlN}$ in wz- and zb-structures, revealing the potential of SL structures in 2D electronics. The objectives of the previous studies on the possible 2D allotropes of GaN and AlN have been to determine their electronic energy bands and hence to reveal their differences from 3D bulk phases. Tuning the electronic structure is possible by stacking different numbers of layers, applying strain or by the decoration of adsorbed foreign atoms. A large number of data collected from these studies emphasize that SL and ML structures of GaN and AIN keep the promise of future electronic materials.

The $\pi$ and $\pi^{*}$-bands lead to a fundamental band gap of $\mathrm{h}-\mathrm{GaN}$, making it a wide band gap semiconductor. As can be seen in Fig. 3, maximum of the valence band occurring at the K-point and minimum conduction band at the $\Gamma$-point yield an indirect PBE band gap of $E_{g-i}=2.16 \mathrm{eV}$, which is significantly larger than the band gap of 3D wz(zb)-GaN calculated by $\mathrm{PBE}$ as $E_{g-d}=1.71$. Upon correction with HSE and $G_{0} W_{0}$ methods, band gaps of h-GaN were obtained as 3.42 and $4.55 \mathrm{eV}$, respectively. ${ }^{34}$ Spin-orbit coupling (SOC) 
TABLE IV. Free-standing, SL h-AlN, t-AlN, and so-AlN: Optimized lattice constant $a$; Al-N bond length $d$ (two different Al-N bond lengths $d_{1} / d_{2}$ for soAlN), cohesive energy $E_{c}$ per Al-N pair; in-plane stiffness $C$ (uniaxial strain along $x$-axis/along diagonal of $\sqrt{2} \times \sqrt{2}$ of so-AlN), Poisson's ratio $\nu$ (uniaxial strain along $x$-axis/along diagonal of $\sqrt{2} \times \sqrt{2}$ cell of so-AlN), charge transfer $Q_{b}^{*}$ from Al to N, Born effective charge $Z^{*}$, and indirect $E_{g-i}$ and direct $E_{g-d}$, band gaps calculated using different methods.

\begin{tabular}{|c|c|c|c|c|c|c|c|c|c|c|c|}
\hline & & $\begin{array}{c}a \\
(\AA)\end{array}$ & $\begin{array}{c}d \\
(\AA)\end{array}$ & $\begin{array}{c}z \\
(\AA)\end{array}$ & $\begin{array}{c}E_{c} \\
(\mathrm{eV} / \mathrm{AlN})\end{array}$ & $\begin{array}{c}C \\
(\mathrm{~N} / \mathrm{m})\end{array}$ & $\begin{array}{c}\nu \\
(\%)\end{array}$ & $\begin{array}{l}Q_{b}^{*} \\
\text { (e) }\end{array}$ & $\begin{array}{l}Z^{*} \\
\text { (e) }\end{array}$ & $\begin{array}{l}E_{g-i} \\
(\mathrm{eV})\end{array}$ & $\begin{array}{l}E_{g-d} \\
(\mathrm{eV})\end{array}$ \\
\hline $\mathrm{h}$ & PBE/HSE & 3.13 & 1.81 & - & 10.56 & 114 & 0.46 & 2.32 & 2.73 & $2.91 / 4.00$ & $3.62 / 4.84$ \\
\hline $\mathrm{h}$ & $\mathrm{LDA}^{20}$ & 3.09 & 1.79 & - & 14.30 & 116 & 0.46 & 0.73 & - & $3.08\left(G W_{0}: 5.57\right)$ & - \\
\hline $\mathrm{h}$ & $\mathrm{PBE}^{32}$ & 3.13 & 1.81 & - & 10.72 & - & - & 2.28 & - & 2.92 (HSE:4.06) & 3.62 \\
\hline $\mathrm{h}$ & $\mathrm{PBE}^{115}$ & 3.17 & 1.83 & - & 10.10 & - & - & - & - & 2.88 & - \\
\hline $\mathrm{h}$ & $\mathrm{PBE}^{30}$ & 3.13 & - & - & - & - & - & 2.29 & 2.71 & (HSE:4.85/ $G_{0} W_{0}: 5.03$ ) & - \\
\hline $\mathrm{h}$ & $\mathrm{PBE}^{116}$ & 3.13 & - & - & - & - & - & - & - & 2.91 & - \\
\hline $\mathrm{h}$ & $G_{0} W_{0}^{114}$ & 3.03 & - & - & - & - & - & - & - & 5.8 & 6.5 \\
\hline $\mathrm{t}$ & PBE/HSE & 3.63 & 1.90 & 0.56 & 10.42 & 70 & 0.52 & 2.42 & 3.01 & $3.55 / 4.75$ & $3.97 / 5.26$ \\
\hline $\mathrm{t}$ & $\mathrm{PBE}^{116}$ & 3.61 & - & 0.58 & - & - & - & - & - & 3.69 & - \\
\hline $\mathrm{t}$ & $\mathrm{PBE}^{30}$ & 3.61 & - & - & - & - & - & - & - & - & - \\
\hline so & $\mathrm{PBE}^{109}$ & 6.15 & $1.83 / 1.76$ & - & 10.04 & $38.22 / 143$ & $0.79 / 0.22$ & - & - & 2.87 (HSE:4.09) & 3.03 \\
\hline so & $\mathrm{PBE}^{117}$ & - & $1.83 / 1.76$ & - & 10.18 & - & - & 3.00 & - & 2.86 & - \\
\hline
\end{tabular}

at the $\Gamma$-point near the top valence band leads to the splitting of the degenerate bands by only $11 \mathrm{meV}$. As for total (TDOS) and orbital projected density of states (PDOS) shown in Fig. 3, they also differ from those of 3D GaN and display the strong peaks due to the flattening of the bands near the conduction band edge. Prete et al. ${ }^{114}$ have recently calculated the bands of $2 \mathrm{D}$ nitrides using the $G W$ approach, where h-GaN yields a direct gap of 4.10 at $\Gamma$ and h-AlN an indirect band gap of $5.8 \mathrm{eV}$ from $\mathrm{K}$ to $\Gamma$. Moreover, by alloying 2D nitride structures, they have tailored the electronic and optical properties of the heterostructures. Band gap tailoring of 2D SL GaN is also possible via decorating with $\mathrm{H}$ or $\mathrm{F}$ adatoms or applying external electric field, as demonstrated by Chen et al. ${ }^{26}$

h-AlN was found to have an $E_{g-i}$ of $2.91 \mathrm{eV}$ using PBE, between the $\mathrm{K}$ and $\Gamma$ points in Fig. 4 , according to the new Density Functional Theory (DFT) calculations. $E_{g-i}$ increased to $4.0 \mathrm{eV}$ after the HSE correction. SOC gives rise to a splitting of only $0.6 \mathrm{meV}$ at the $\Gamma$ point at the top valence band. The fundamental band gap of t-AIN, as seen in Fig. 4, was calculated using PBE to be indirect and $E_{g-i}=3.55 \mathrm{eV}$, between the valence band around midpoint of $\Gamma$ and $\mathrm{X}$-points and the conduction band at the $\Gamma$ symmetry point. Similarly, SOC effect was minimal with a negligible splitting at the $\Gamma$ point at the top of the valence band. Moreover, the band gap correction by HSE increased the gap up to $4.75 \mathrm{eV}$. In contrast to h$\mathrm{GaN}$, the fundamental band gaps of 2D AlN phases both appear smaller than those of 3D AIN phases calculated by $\mathrm{PBE}$ as $E_{g-d}=4.21$ for $3 \mathrm{D} \mathrm{wz}-\mathrm{AlN}$ and $E_{g-i}=3.28 \mathrm{eV}$ for 3D $\mathrm{zb}-\mathrm{AlN}$. This situation is contrary to what is encountered in most other 2D structures. Electronic properties of single-layer and few-layer h-AlN were also investigated previously in several other studies. ${ }^{20,30,114,116}$

The fundamental band gaps of so-GaN and so-AlN in Fig. 5 are both indirect and are calculated by PBE as $E_{g-i}=1.85$ and $2.78 \mathrm{eV}$, respectively. ${ }^{109}$ Notably, these band gaps of sostructures come out smaller than that of h-GaN and h-AlN. The HSE correction to the PBE band gaps raises the fundamental band gaps to 3.37 and $4.09 \mathrm{eV}$. The calculated and experimental values related with the band gaps of all 2D allotropes of GaN and AIN are presented in Tables III and IV, respectively.

\section{Effect of strain}

The electronic properties of SL h-GaN under the applied biaxial in-plane tensile strain, $\epsilon$, have been extensively investigated earlier, ${ }^{34}$ which could be crucial for tuning of the electronic structure. The band gap of h-GaN was found to decrease monotonically from 2.16 to $0.21 \mathrm{eV}$, until $10 \%$ strain, even to close at $\epsilon=16 \%$. This is a significant change in the electronic structure caused by the applied strain, in case strain $\epsilon>10 \%$ is affordable for $\mathrm{h}-\mathrm{GaN}$. A recent study on strain engineering of few-layer $\mathrm{GaN}$ showed that the haeckelite atomic structure of layered GaN transforms to planar under applied in-plane tensile strain, while compressive strain induces a transformation into (an increased) direct gap. ${ }^{119}$

Regarding ultrathin AlN, the graphitic hexagonal phase was shown to be a more favorable configuration only for small thicknesses. ${ }^{21}$ On the other hand, stable hexagonal structure for larger thicknesses can still be preserved under epitaxial strain. ${ }^{29}$ Kecik et al. ${ }^{33}$ investigated the modification of the optoelectronic properties of SL and few-layer h-AlN under the influence of in-plane tensile strain at values of $1 \%$, $3 \%, 5 \%$, and $7 \%$. Accordingly, the optical absorption spectra were observed to be globally shifted toward lower photon energies. The absorption band edges, as well as peak positions were redshifted. These shifts were rather uniform and increasing upon higher strain values. Moreover, bond length affected by tensile strain as well as confinement effects are expected to also modify the electronic structure of h-AIN. Hence, band gap of h-AlN was reduced monotonically as the strain value increased.

Tuning of the atomic structure, electronic, and optical properties by in-plane tensile or compressive strain is wellknown also from few recent works on the structural, electronic, and optical properties of 2D materials and group-III nitrides and promising for potential novel optoelectronic applications by strain engineering. ${ }^{29,79,119,120}$ 

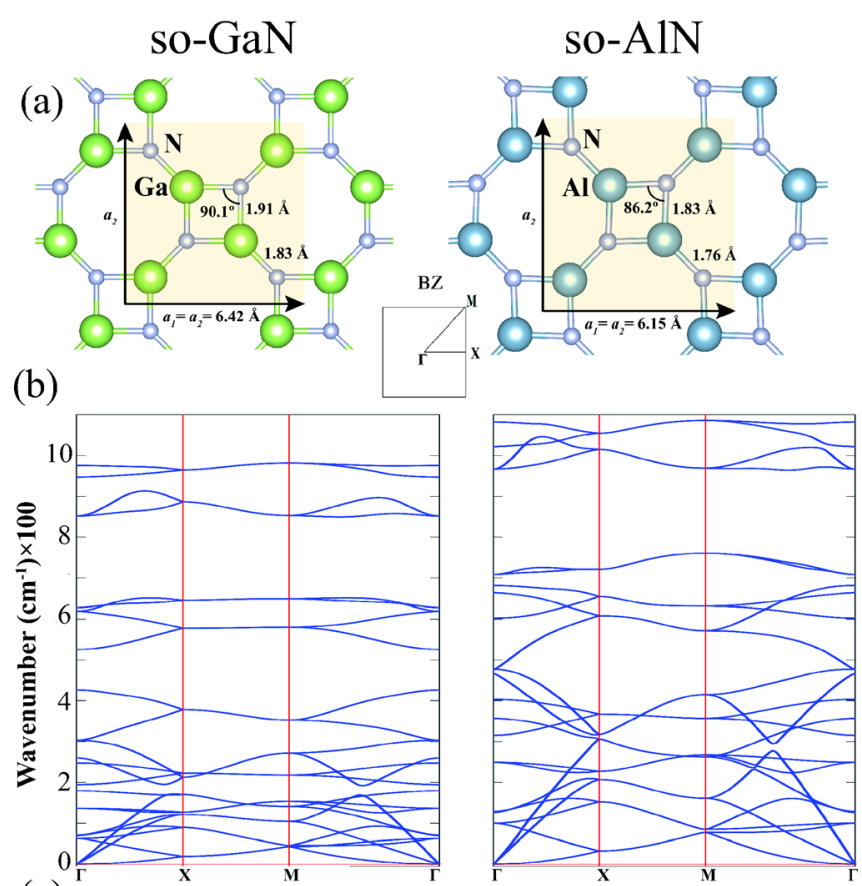

(c)
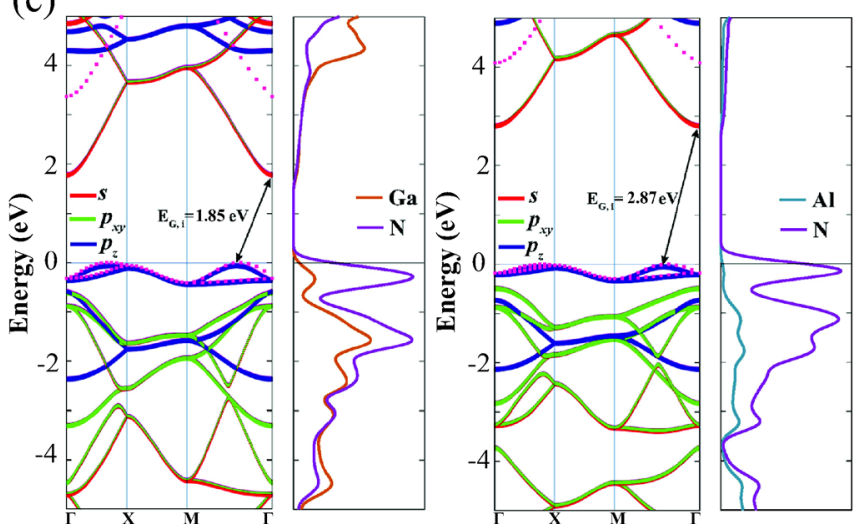

FIG. 5. (a) Top views of the atomic structure of square-octagon, so-GaN, and so-AlN. The primitive unit cell of $2 \mathrm{D}$ square lattices is indicated. (b) Calculated phonon dispersion curves along major symmetry directions. (c) Electronic energy band structures together with the PDOS of $\mathrm{Ga}(\mathrm{Al})$ and $\mathrm{N}$ atoms. Indirect fundamental band gaps $E_{g-i}$ are indicated by arrows. Zero of energy is set to the top of the valence band. PBE bands corrected by HSE are shown by the dashed lines. Reproduced with permission from Gürbüz et al., Phys. Rev. B 96, 205427 (2017). Copyright 2017 American Physical Society.

\section{Effect of substrate}

The freestanding h-GaN cannot be exfoliated from 3D layered $\mathrm{GaN}$, since the latter is nonexistent in nature. Therefore, $2 \mathrm{D} \mathrm{GaN}$ should be grown on a substrate. It is critical to reveal whether the mechanical and electronic properties discussed above for free-standing SL GaN and AlN will survive when these materials are grown on specific substrates. To this end, the structural and electronic properties of SL h-GaN ${ }^{34}$ and h-AlN overlayers grown on two different substrates were examined, which are (i) metallic $\mathrm{Al}(111)$ surface and (ii) semiconducting blue phosphorene. The models of h-GaN + substrate and h-AlN + substrate are presented in Fig. 6.

The first type of substrate, a reactive $\mathrm{Al}(111)$ surface is expected to interact strongly with h-GaN, constructed of a (a)

(b)

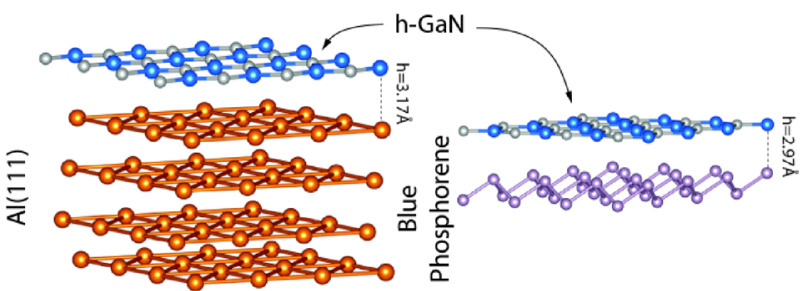

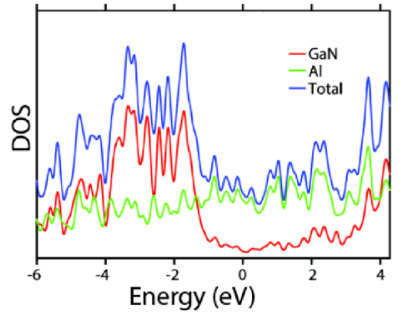

(c)

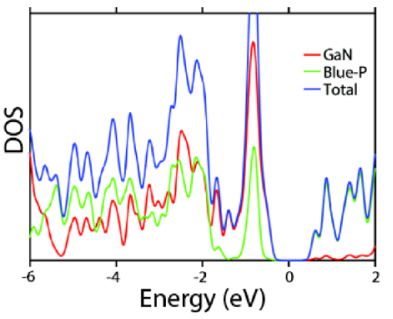

(d)
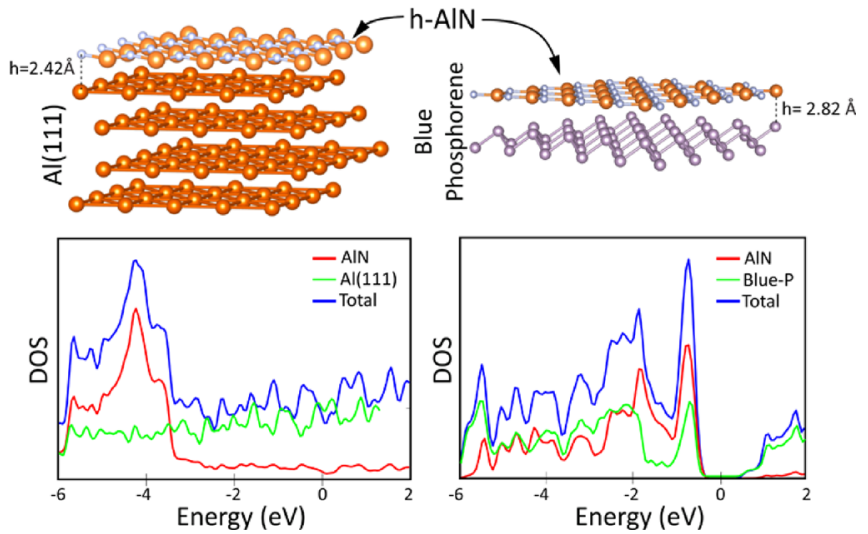

FIG. 6. (a) Optimized atomic structure of h-GaN overlayer on $\mathrm{Al}(111)$ slab represented by four $\mathrm{Al}(111)$ atomic planes together with the calculated total and local densities of states on the overlayer as well as on $\mathrm{Al}(111)$ slab. (b) Optimized atomic structure of h-GaN overlayer on a SL blue phosphorene together with the calculated total and local densities of states on the overlayer as well as on SL blue phosphorene. Parts (a) and (b) of this figure are reproduced with permission from Onen et al., Phys. Rev. B 93, 085431 (2016). Copyright 2016 American Physical Society. (c) Same as (a) for hAlN. (d) Same as (b) for h-AlN. Parts (c) and (d) of this figure are based on complementary new data for the sake of comparison.

slab consisting of four $\mathrm{Al}(111)$ planes. In order to ensure lattice matching with h-GaN overlayer, $\mathrm{Al}(111)$ slab has been expanded by $15 \%$, so that the $\mathrm{N}$ atoms are located on top of the $\mathrm{Al}$ atoms. The optimized height of $\mathrm{h}-\mathrm{GaN}$ from the substrate is $h=3.17 \AA$, larger than the sum of the covalent atomic radii, $r_{G a}+r_{N}=1.76 \AA .{ }^{121}$ Density of states (DOS) localized on the overlayer reveals peaks $1<E<2 \mathrm{eV}$ and $3<E<4 \mathrm{eV}$. Low DOS near the gap region of $\mathrm{h}-\mathrm{GaN}$ in the energy range $1<E<1.5 \mathrm{eV}$ are partly due to numerical accuracy and weak substrate-overlayer interaction.

Blue phosphorene, a semiconducting buckled 2D material is nearly lattice matched to h-GaN, hence is an ideal substrate to investigate the substrate-overlayer interaction. As shown in Fig. 6(a), the height of the h-GaN overlayer from blue phosphorene surface is $h=2.97 \AA$, larger than the sums of covalent radii $r_{G a}+r_{P}=2.36 \AA$. The DOS projected onto $\mathrm{h}-\mathrm{GaN}$ displays peaks at $\sim-1 \mathrm{eV},-2.5 \mathrm{eV}$, and $-6 \mathrm{eV}$. As can be observed, the fundamental band gap of $\mathrm{h}$ $\mathrm{GaN}+$ phosphorene partly overlaps with that of pristine $\mathrm{h}-$ 
$\mathrm{GaN}$, suggesting that the overlayer h-GaN and the underlying blue phosphorene interaction does not modify the electronic structure of the freestanding h-GaN significantly.

Likewise, the effect of $\mathrm{Al}(111)$ and blue phosphorene substrates on h-AlN overlayers is weak as seen in Figs. 6(c) and 6(d). Similarly, the structure, interlayer interaction energy, and electronic properties of SL and few-layer h-AlN on graphene were also investigated within DFT. ${ }^{115}$ It was found that dynamically stable few-layer h-AlN can form on graphene, where interlayer interaction between h-AlN layers is stronger than that between h-AlN and graphene. Also, the electron density of h-AlN layers is not significantly modified upon stacking. A recent study ${ }^{111}$ investigated the properties of h-AlN on $\mathrm{MoS}_{2}$, and claimed that either by inducing vacancies or doping, the physical properties and band alignment type of the AlN/MoS ${ }_{2}$ heterostructure can be adjusted.

\section{MULTILAYERS OF SL-GaN AND SL-AIN}

As several previous works have shown, the modification of the physical properties of layered structures as the layer number is increased is rather gradual. ${ }^{122,123}$ Growing these $\mathrm{BL}, \mathrm{TL}$, or ML, and eventually 3D periodic layered structures, which correspond to local minima on BO surface, can lead to novel artificial materials. ${ }^{124}$ The BL and ML constructions of SL GaN and AlN with weak interlayer interactions have a bearing on the formation of vertical composite structures, heterostructures and Schottky barriers, and hence are closely related with nanodevice fabrication. Here the crucial question to be addressed is whether BL and few-layer structures can be constructed by simply vertical stacking of SL GaN or AlN with weak van der Waals (vdW) interlayer interaction. Since neither 3D GaN nor AlN have a layered structure like graphite, whether ML structures undergo a structural transformation by forming strong vertical chemical bonds to eliminate weakly bound layered structure, has been a focus of curiosity. Additionally, whether these vertical bonds can induce buckling in otherwise planar SL GaN and SL AlN constituents has been a subject of interest. In fact, studies have demonstrated that such a situation may occur for the ML formation from the vertical stacking of silicene, whereby vertical and strong $\mathrm{Si}-\mathrm{Si}$ covalent bonds between layers hinder the formation of the vdW solid. ${ }^{123}$ In this section, the formation of the layered vdW solid from SL GaN and AlN will be reviewed, where also the structural and electronic properties of few-layer $\mathrm{GaN}$ and $\mathrm{AlN}$ will be discussed.

First ML formation has been explored by considering different stacking geometries of both SL h-GaN and h-AlN. The optimum stacking geometries (i.e., energetically most favorable ones) for BL and TL h-GaN were found to $\mathrm{be}^{34}$ $\mathrm{AA}^{\prime}$ (i.e., hexagons on top of each other with $\mathrm{Ga}$ atoms above $\mathrm{N}$ ) and $\mathrm{AA}^{\prime} \mathrm{A}$, respectively. Both stacking geometries are also in agreement with those of $\mathrm{Xu}$ et al. ${ }^{107}$ The corresponding cohesive energies per lateral $\mathrm{Ga}-\mathrm{N}$ pair are $E_{C}=8.57 \mathrm{eV}$ and $E_{C}=8.69 \mathrm{eV}$, respectively. The interlayer interaction energies (where $E_{i}=\left(n \times E_{T}[\mathrm{SLGaN}(\mathrm{AlN})]\right.$ $\left.-E_{T}[\mathrm{MLGaN}(\mathrm{AlN})]\right) / n$ with $n$ single-layers, relative to the constituent SL GaN or AlN) of BL and TL constructed from h-GaN are relatively weak and are calculated to be $E_{i}=284 \mathrm{meV}$ and $E_{i}=399 \mathrm{meV}$ per vertical Ga-N pair, respectively. Notably, owing to the increasing interlayer interaction, the $E_{C}$ of TL h-GaN is larger than that of BL h$\mathrm{GaN}$ and SL h-GaN. The vdW interaction is not taken into account within $3 \mathrm{D}$ bulk structures, hence the bulk $E_{C}$ are slightly underestimated relative to those of the ML structures. The designed 3D layered $\mathrm{GaN}$ structure constituted from planar SL h-GaN is periodic in the direction perpendicular to the atomic planes and has an optimum stacking sequence of $\mathrm{AA}^{\prime} \mathrm{AA}^{\prime} \ldots$ with $E_{c}=8.94 \mathrm{eV}$ and the interlayer interaction energy $E_{i}=653 \mathrm{meV}$ per vertical Ga-N pair.

The interlayer spacing $h$, lattice constants $a=b, c$ and bond length $d$ along with the corresponding electronic structures of $\mathrm{BL}, \mathrm{TL}$, and $3 \mathrm{D}$ periodic $\mathrm{h}-\mathrm{GaN}^{34}$ are shown in Fig. 7(a). Clearly, the interlayer interaction energies in $\mathrm{BL}$ and $\mathrm{TL}$ are dominated by the $\mathrm{vdW}$ interaction, since significant chemical bonding cannot set at the interlayer distances in the vicinity of $2.5 \AA$. However, 3D periodic layered structure with an interlayer distance of $2.44 \AA$ attains slight chemical interlayer interaction. The indirect band gap of SL h-GaN decreases to $1.98 \mathrm{eV}$ in $\mathrm{BL} \mathrm{h}-\mathrm{GaN}$ and to $1.83 \mathrm{eV}$ in TL h$\mathrm{GaN}$. Moreover, $h$ also decreases as the number of layers increases, since the total interlayer interaction also increases, contrary to the trend of bond lengths and lattice constants. In $3 \mathrm{D}$ periodic $\mathrm{h}-\mathrm{GaN}$, on the other hand, where the total interlayer interaction is maximum, the fundamental band gap decreases to $1.23 \mathrm{eV}$ and becomes direct. This trend can be explained in terms of the confinement effect. Notably, the band gap of 3D wz- and zb-GaN is direct, yet significantly larger than that of 3D periodic $\mathrm{h}-\mathrm{GaN}$. This is an important result and shows that 3D layered structure of h-GaN can be synthesized with a band gap smaller than those of 3D bulk crystals. Additionally, the band gap of few-layer h-GaN decreases with increasing number of layers and changes from indirect to direct. Hence, the number of layers can be a controllable structural parameter for tuning the electronic properties. The band gap character and value of few-layer hGaN can also be tuned by changing the stacking order, as well as by applying electric field. ${ }^{107}$

Likewise, for the ML structures of h-AlN, BL, TL, and 3D bulk periodic forms of h-AlN were examined in terms of their structural parameters, cohesive energies, electronic, and optical properties in the previous studies. ${ }^{32,33}$ Similar to the stacking of ML of h-GaN, the favorable stacking sequence for $\mathrm{BL}, \mathrm{TL}$, and 3D layered h-AlN were found to be $\mathrm{AA}^{\prime}, \mathrm{AA}^{\prime} \mathrm{A}$, and $\mathrm{AA}^{\prime} \mathrm{AA}^{\prime} \ldots$... respectively, as shown in Fig. 7(b). The same trends discussed above for ML h-GaN were also obtained for $\mathrm{BL}, \mathrm{TL}$, and 3D layered periodic structures of h-AIN, in terms of the decrease in interlayer spacing and increase in bond length with the increasing number of layers. Moreover, the stability of bilayer structures of both h-GaN and h-AlN in honeycomb symmetries with an $\mathrm{AA}^{\prime}$ stacking has been confirmed by phonon frequency calculations. Regarding the gradual changes in the electronic properties, the indirect band gap of SL h-AlN increases to $3.54 \mathrm{eV}$ in BL as well as in TL hAlN. This has been confirmed in Ref. 32, which refers to the 
(a)
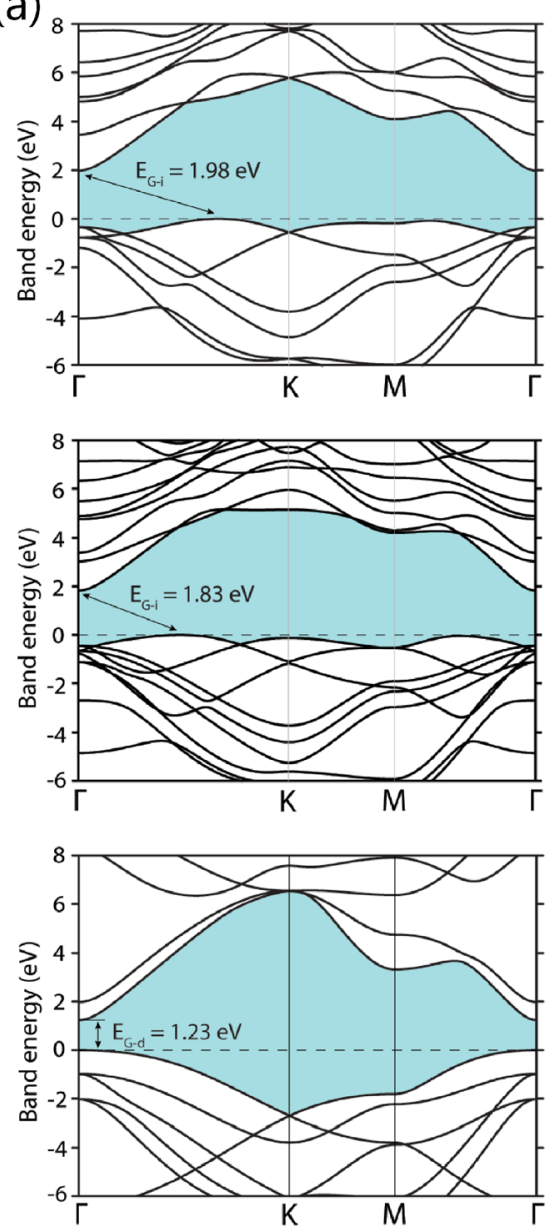

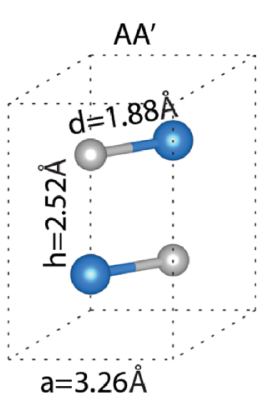

(b)
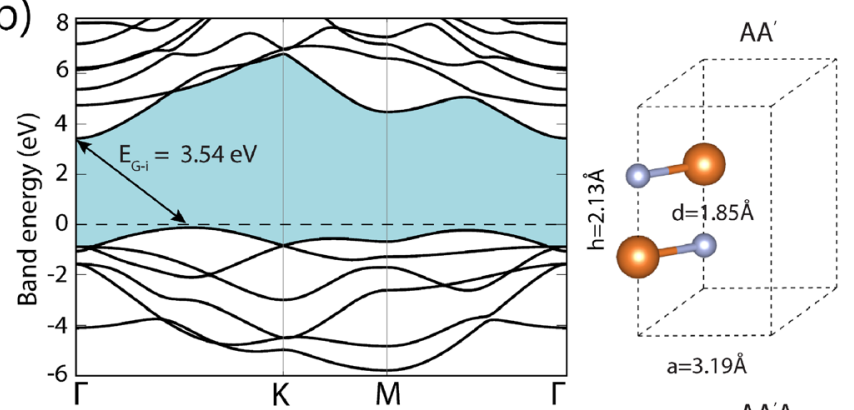

'AA'A

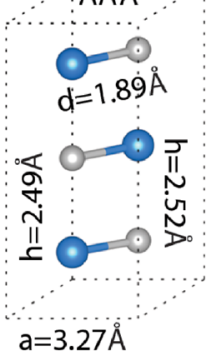

$A A^{\prime} A A^{\prime} .$.

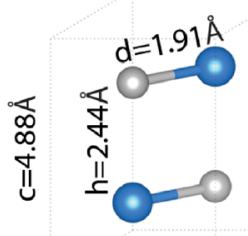

$\mathrm{a}=\mathrm{b}=3.31 \AA$
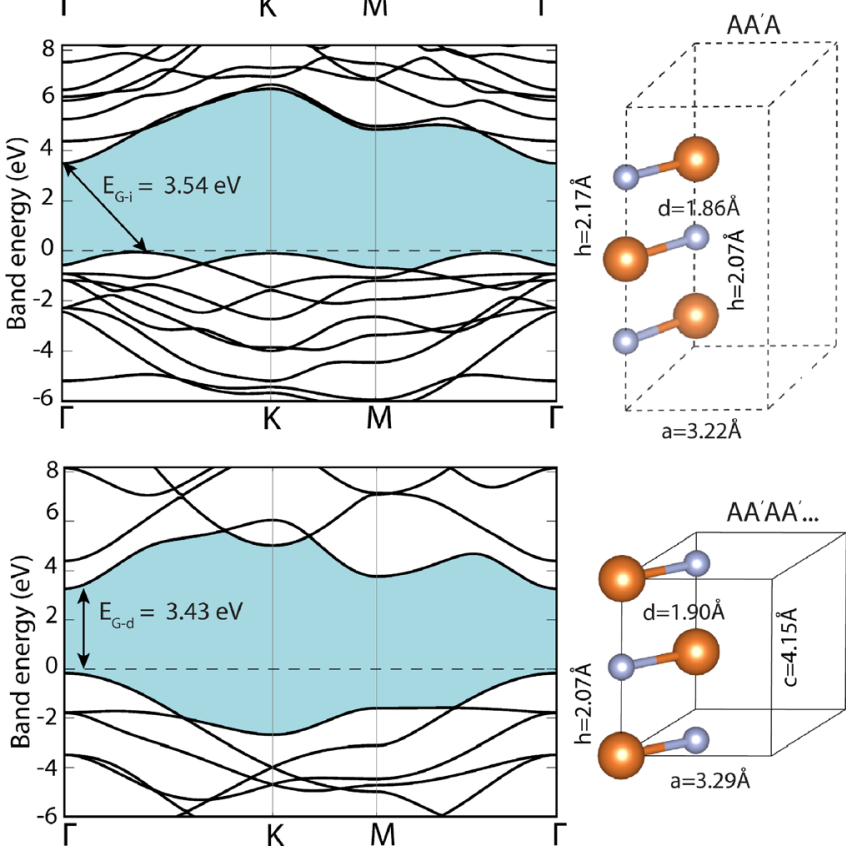

FIG. 7. (a) Energy band structures of few-layer h-GaN: (top) BL h-GaN with optimized AA' stacking; (middle) trilayer (TL) h-GaN with AA'A stacking; and (bottom) 3D periodic layered structure of h-GaN with $\mathrm{AA}^{\prime} \mathrm{AA}^{\prime}$... stacking. This part is reproduced with permission from Onen et al., Phys. Rev. B 93, 085431 (2016). Copyright 2016 American Physical Society. (b) Energy band structures of few-layer h-AlN: (top) BL h-AlN with optimized AA' stacking; (middle) TL h-AlN with AA'A stacking; and (bottom) 3D periodic layered structure of h-AlN with $\mathrm{AA}^{\prime} \mathrm{AA}^{\prime}$... stacking. Part (b) is based on new data provided for the sake of comparison and complementing existing results. The primitive unit cell is delineated by dashed lines. Zero of energy is set to the top of the valence bands. Fundamental band gaps are shown by arrows. Interlayer distances $h$ and interatomic bonds $d$ are indicated.

band gap of BL h-AlN as indirect and $3.5 \mathrm{eV}$. Finally, the band gap of 3D periodic structure of h-AlN is predicted to be direct and decreased to $3.43 \mathrm{eV}$, namely, smaller than the direct band gap of 3D wz-AlN and larger than the indirect band gap of 3D zb-AlN. Although 3D layered and periodic hAlN are direct gap insulators, it was recently shown that its layered structure reveals an indirect gap character up to ten layers. ${ }^{32}$ The increase of the fundamental band gap in BL and TL is surprising and is in contrast with the confinement effects.

Similar to ML structures constructed from $\mathrm{h}-\mathrm{GaN}$ and $\mathrm{h}-$ AlN, Gürbüz et al. ${ }^{109}$ investigated the binding BL, TL, and 3D periodic layered structures of so-GaN and so-AlN for three stable stacking parameters such as $\mathrm{AA}^{\prime}, \mathrm{AB}$, and cubane. Among these stacking geometries, they found that $\mathrm{AA}^{\prime}$, cubane, and deformed $\mathrm{AB}$ stacking geometries attain minimum total energies for $\mathrm{BL}, \mathrm{TL}$, and $3 \mathrm{D}$ periodic structures, respectively. The interlayer interaction energies of BL, $\mathrm{TL}$, and 3D periodic layered structures constructed from $\mathrm{AA}^{\prime}$-type stacking of so-GaN (so-AlN) are calculated as $400 \mathrm{meV}(620 \mathrm{meV}), 382 \mathrm{meV}(607 \mathrm{meV})$, and $399 \mathrm{meV}$ $(645 \mathrm{meV})$ per vertical cation-anion pair. The interlayer spacing or the length of vertical Ga-N (Al-N) pairs is found to be $2.23 \AA(2.10 \AA)$, which are smaller than those of ML structures of h-GaN (h-AlN). The planar free-standing SL so-GaN and so-AlN structures acquired minute buckling upon vertical stacking. Remarkably, a different structure of $\mathrm{BL}$ in cubane structure which is obtained from $\mathrm{AA}^{\prime}$ stacking with almost the same energy of $390 \mathrm{meV}$ is found, where vertical Ga-N bonds between the squares are shortened, while similar vertical bonds between the octagons were elongated displaying a haeckelite like configuration in the side view.

Motivated with this new configuration, we explored the possibility whether such a haeckelite reconstruction can take place to further increase the cohesive energy of ML structures of planar h-GaN and h-AlN. Recently, Kolobov et al. ${ }^{28}$ have also predicted an interlayer haeckelite configuration of few-layer GaN to be the most stable structure of few-layer GaN (compared to planar and wurtzite-like few-layers). According to our calculations specifically performed to investigate the conditions haeckelite like interlayer bonds form, it was observed that BL of "buckled" or "flat" GaN layers conformed with AA or $\mathrm{AA}^{\prime}$ stacking do not transform 
into a haeckelite structure by themselves. Also, when compared with the interlayer interaction energies of BL, TL, four layer (FL), and 3D periodic structures of planar $\mathrm{h}-\mathrm{GaN}$, the vertical haeckelite like structures of BL, TL, FL, and 3D periodic GaN have energies 5, 96, 164, and $395 \mathrm{meV}$ per Ga$\mathrm{N}$ pair lower than the planar counterparts, which indicate favored stabilities. Notably, there are other allotropes of BL of h-GaN, which are even energetically more favorable than the heackelite structure. As for AlN, haeckelite like BL, TL, and $3 \mathrm{D}$ periodic conformations were not preserved; rather they conformed back to flat-like ML structures, in agreement with Ref. 32.

\section{A. Optical properties of GaN and AIN}

In this section, we will discuss the optical spectra of h$\mathrm{GaN}$ and h-AlN and compare them with their bulk counterparts. Since 3D wz-GaN is well-known for its use in optoelectronic applications, the optical properties of its 2D phases have also been a subject of interest. In Figs. 8(a)-8(d), the optical absorption spectra and layer dependent absorbance of SL and BL h-GaN and h-AlN are presented, all calculated using random phase approximation (PBERPA), based on Refs. 33 and 34. We note that RPA spectra usually differ dramatically from experimental counterparts due to its shortcomings especially pronounced for lowdimensional band gap materials with enhanced many-body effects. Hereby, although the results obtained by BetheSalpeter equation (BSE) method which take into account the electron-hole interactions are not displayed, we report relevant data in the forthcoming discussions.

The critical features observed from the imaginary dielectric function $\left[\varepsilon_{2}(\omega)\right]$ and layer dependent absorbance $[\mathrm{A}(\omega)]$ can be summarized as: (i) The onsets of the optical absorption of SL and BL in $\varepsilon_{2}(\omega)$ are in compliance with each other and also with their band gaps calculated within PBE. (ii) The peak in $\varepsilon_{2}(\omega)$ of h-GaN occurs due to the optical transitions from flat occupied $\pi$-bands between $\mathrm{K}$ and
M-points to flat empty $\pi^{*}$ bands $\sim 5 \mathrm{eV}$ above, which seems to be more spread in $\mathrm{BL} h-\mathrm{GaN}$. (iii) $3 \mathrm{D}$ wz-GaN displays an absorption onset below that of h-GaN and multi-peak features between 5 and $12 \mathrm{eV}$. (iv) The optical absorption for 2D h-GaN kicks-off within the visible light region or more towards the UV range, differently from 3D wz-GaN. (v) The lower amplitude in $\varepsilon_{2}(\omega)$ of h-GaN compared to 3D wz-GaN is due to the weaker linear optical response of a single layer of h-GaN, normal to the incident light. (vi) The so-called layer dependent absorbance reveals that $\mathrm{BL}$ h-GaN naturally displays an elevated overall absorbance in general, with respect to SL h-GaN, reaching a maximum of $\sim 17 \%$.

The results of the extensive study on the optical response of few-layer h-AlN analyzed by means of various optical spectra are reported in Ref. 33, where the influence of advancing first-principles methods, number of layers, and inplane tensile strain were considered. Here, we discuss the optical properties of SL and BL h-AlN. In Figs. 8(b) and $8(\mathrm{~d})$, the $\varepsilon_{2}(\omega)$ and $A(\omega)$ of SL and BL h-AlN are plotted. SL h-AlN's $\varepsilon_{2}(\omega)$ displays an onset at $\sim 3.4 \mathrm{eV}$, where that of BL h-AlN appears around $4.2 \mathrm{eV}$, which are related with the respective band gaps of 2.91 and $\sim 3.5 \mathrm{eV}$, the latter approaching to the absorption onset of bulk h-AlN $(4.5 \mathrm{eV})$. An intense absorption occurring for layered h-AlN is observed in the range of $6.8-10.8 \mathrm{eV}$, indicating that more layers are required to attain a bulk-like character. Moreover, SL h-AlN exhibits a single distinct absorption peak around $6 \mathrm{eV}$, related with the significant transitions at the $\Gamma, \mathrm{M}$, and $\mathrm{K}$ points. On the other hand, BL h-AlN displays rather scattered multi-peaks, first one around $5.4 \mathrm{eV}$, second near $7.2 \mathrm{eV}$ and third one above $9.4 \mathrm{eV}$. The increased absorbance intensities upon increased number of layer is a repeated pattern for h-AlN, similar to h-GaN.

Regarding the many-body effects on the optical properties, we refer to the earlier works ${ }^{33,34}$ for h-GaN and h-AlN, respectively. In general, it was observed that HSE and $G_{0} W_{0}$ methods blueshifted the absorption onsets as well as the

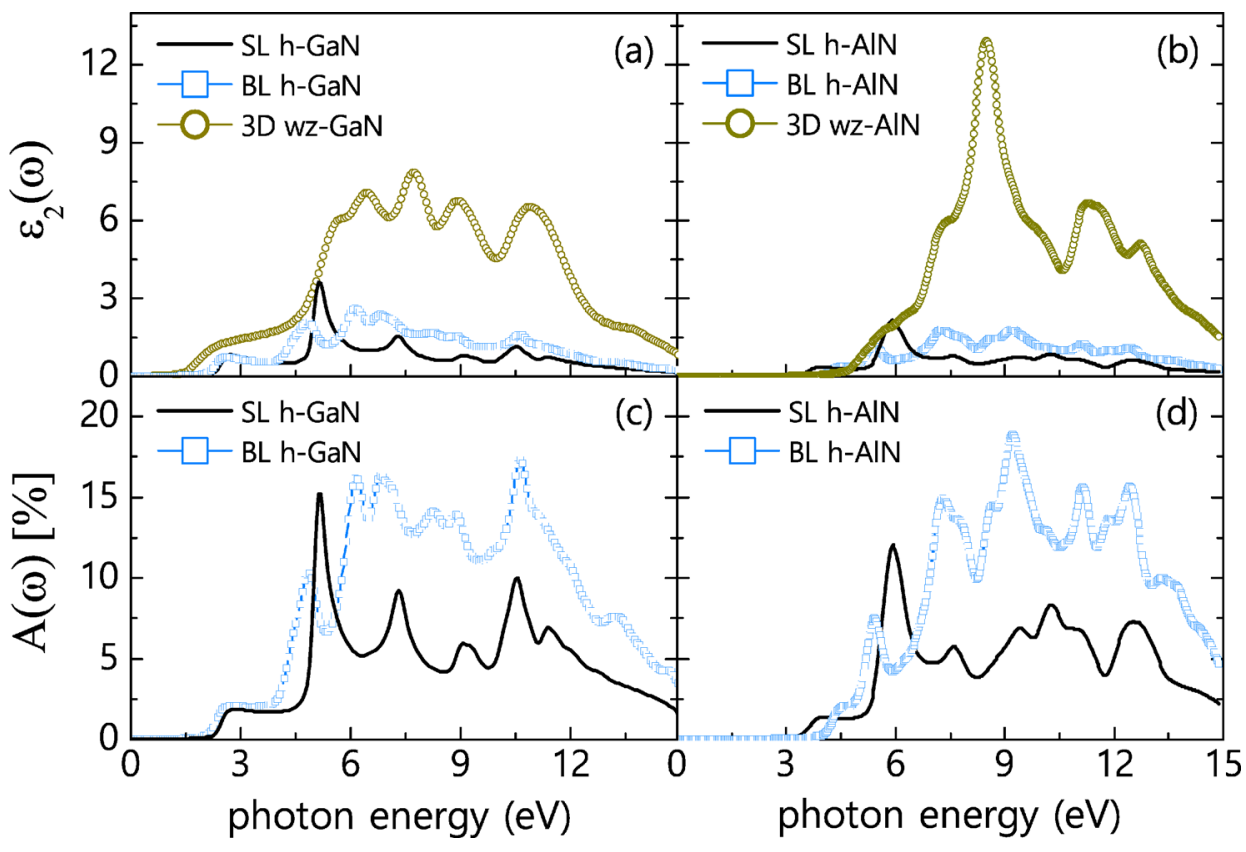

FIG. 8. Optical absorption spectra, i.e., $\varepsilon_{2}(\omega)$ calculated using PBE-RPA of (a) SL, BL h-GaN, and 3D wz-GaN, and (b) SL, BL h-AlN, and 3D wz-AlN. Layer dependent absorbance, $\mathrm{A}(\omega)$, versus photon energy of (c) SL and BL $\mathrm{h}-\mathrm{GaN}$ and (d) SL and BL h-AlN. Reproduced with permissions from Kecik et al., Phys. Rev. B 92, 165408 (2015) and Onen et al., Phys. Rev. B 93, 085431 (2016). Copyright 2015 and 2016 American Physical Society. 
overall spectra. Absorption onset energy obtained from PBE calculations was blueshifted for h-GaN by $\sim 1.5$ and by $1 \mathrm{eV}$ for h-AlN, once HSE corrections were applied. Moreover, strong exciton binding was observed for the latter structure, due to the electron-hole interactions originated from weak screening in wide-gap low-dimensional AlN, similar to the results in a recently published work ${ }^{31}$ where a bright exciton binding energy was found as $2.05 \mathrm{eV}$. Binding energies for other 2D materials were found as $0.8 \mathrm{eV},{ }^{125}$ close to $1 \mathrm{eV},{ }^{126}$ and $2.1 \mathrm{eV}^{127}$ for monolayer phosphorene, $\mathrm{MoS}_{2}$ and $\mathrm{BN}$, respectively. The exciton binding energy of h-AlN is substantially higher than that of its 3D wurtzite structure $(1 \mu \mathrm{m}$ thick film), measured as $80 \mathrm{meV}^{128}$ and AlN films, of $63 \mathrm{meV} .{ }^{129}$ Seminal important works on the optical properties and excitonic effects within bulk AlN have also been performed recently. ${ }^{130,131}$

The optical spectra of SL and bulk GaN and AlN show significant differences, implying that they could serve for different optoelectronic applications. As it has been discussed in Sec. IV, the electronic properties of BL and fewlayer structures of $\mathrm{h}-\mathrm{GaN}$ and $\mathrm{h}$-AlN also undergo gradual changes with the number of layers. Therefore, one can control the optical properties by changing the number of layers in the ML structures.

Recently, Attaccalite et al. ${ }^{113}$ have performed firstprinciples calculations of the second harmonic generation intensity spectrum of $2 \mathrm{D} \mathrm{GaN}$. Via $G_{0} W_{0}$ calculations, they found that SL GaN has a direct band gap of $4.27 \mathrm{eV}$, whereas the LDA gap is indirect and yields $2.36 \mathrm{eV}$. The results for the gap are close to those reported by Chen $\mathrm{et} \mathrm{al.} .^{26}$ and comparable to that calculated as $4.55 \mathrm{eV}$ by Onen et al. ${ }^{34}$ using $G_{0} W_{0}$. Moreover, excitonic peaks appeared at 3.1 and $4.8 \mathrm{eV}$ in the $G_{0} W_{0}$-BSE spectrum of SL GaN, revealing an exciton binding energy in agreement with that for sheet-like GaN. ${ }^{132}$ The reader may refer to Ref. 133 for a comprehensive review of the excitonic properties of atomically thin 2D materials and layered vdW heterostructures, comprising TMDs, graphene, phosphorene, and h-BN.

\section{COMPOSITE STRUCTURES OF GaN/AIN}

Earlier, a significant portion of the 3D optoelectronic nitride devices were fabricated in the form of heterostructures, where numerous theoretical studies are also present. ${ }^{134-136}$ Composite structures made of lateral and vertical junctions of 2D SL structures like Graphene/BN or $\mathrm{MoS}_{2} /$ $\mathrm{WS}_{2}$ that have been previously treated both theoretically and experimentally, heralded great potential for new generation artificial structures with promising applications. ${ }^{20,137-141}$ Motivated with the synthesis of very thin h-GaN and h-AlN, Onen et $a l .{ }^{35}$ recently studied the composite structures of their single-layers. They showed that stable, in-plane composite materials, $(\mathrm{GaN})_{p} /(\mathrm{AlN})_{q}$ can be constructed of periodically repeating stripes of GaN and AlN continuously (or commensurately) joined along their zigzag (Z) edges. Here, $p$ and $q$, which denote the numbers of formula units of the constituent compounds in the unit cell, are controllable parameters. These composite materials exhibit a wide range of physical properties depending on the values of $p$ and $q$ : A structure having $p \simeq 1-2$, but very large $q$, or vice versa, is identified as a $\delta$-doping, where very narrow ribbon of $\mathrm{GaN}$ is implemented commensurately in a large AlN stripe, or vice versa. For $p=1-2$, and $q=1-2$, the composite structure behaves differently from its parent constituents as if a compound of lines of GaN and AlN. For small $p$ and $q$, the fundamental band gap of the composite can be tuned by varying $p$ and $q$. On the other hand, for large $p$ and $q$, the electronic states become confined to one of the constituent stripes, experiencing a transition from $2 \mathrm{D}$ to $1 \mathrm{D}$. At the boundary region of the junction, charge is transferred from one region to another and normally sets the band-lineup, constructing multiple quantum wells and quasi $1 \mathrm{D}$ quantum structures in a $2 \mathrm{D}$ atomically thin nanomaterial with diverse functionalities. Furthermore, 2D patterned structure of SL GaN quantum dots can be implemented to 2D SL h-AlN to form periodically arranged quantum dots. In Fig. 9, the composite structure, a finite size heterostructure comprising single quantum well, and a regular 2D patterned core/shell array constructed from SL GaN/AIN are displayed.

The properties of 2D in-plane or vertical composites of GaN/AlN or GaN with another 2D material have been vastly studied over the past few years. ${ }^{142-144}$ For example, Chen et al. ${ }^{142}$ studied 2D lateral GaN/SiC heterostructure within DFT and deduced six inequivalent interfaces in different heterostructures where they predicted strong magnetoelectric coupling and interface magnetization related to the density of states at the Fermi level due to Stoner instability.

Vertical heterostructures of h-GaN and h-AlN forming a commensurate periodic or single junction are also of interest, since the fundamental band gaps of both constituents are significantly different and can give rise to pronounced bandlineup values. Since the band gaps of both constituents change from indirect to direct and even their values, by themselves, show dramatic variations with the number of layers in the heterostructure, vertical layered $(\mathrm{GaN})_{p} /(\mathrm{AlN})_{q}$ junctions are expected to display rather interesting features. Not only vertical GaN/AIN junctions but also vertical layered GaN/graphite, AlN/graphite or vertical GaN(AIN)/thin metal junctions can form a Schottky barrier at the interface showing rectifying properties.

\section{DISCUSSIONS AND CONCLUSIONS}

Initially, the main concerns of the pioneering studies in this field have been to reveal whether single-layer or ultrathin 2D GaN and AlN compounds can form stable structures like graphene. With theoretical predictions of various stable SL allotropes of these compounds based on extensive analyses of energetics, dynamical, and thermal stabilities, research interests focused on revealing whether the mechanical, electronic, and optical properties of 2D GaN and AlN differ from those of the 3D GaN and AlN crystals. The following critical questions have motivated several theoretical works: Are the properties of SL GaN and AIN relevant for various applications? How do the properties of SL structures change, for example, in BL and ML? How are their electronic structures modified by adsorbing or substituting adatoms, creating defects, or applying strain? Since GaN and AlN are nearly 


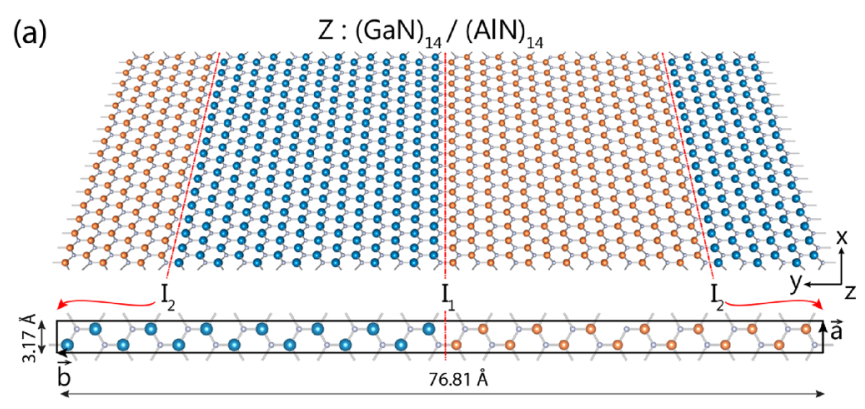

(b)
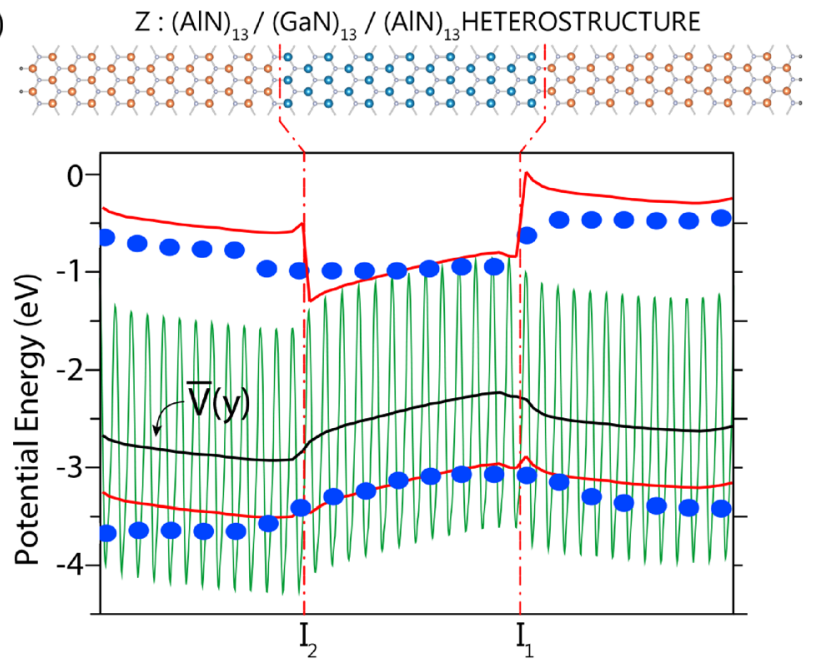

(c)
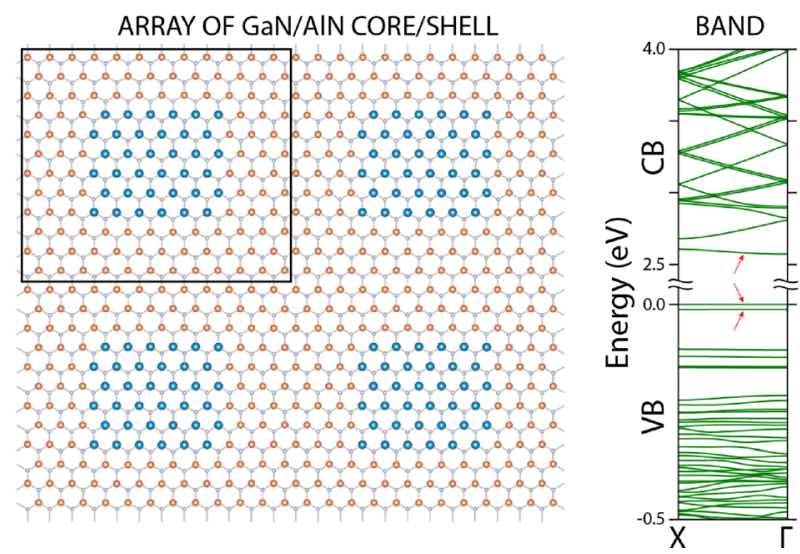

FIG. 9. (a) A schematic description of the atomic structure of $\mathrm{Z}:(\mathrm{GaN})_{p} /$ $(\mathrm{AlN})_{q}$ together with the unit cell and lattice constants. (b) Z:(AlN) $)_{13} /$ $(\mathrm{GaN})_{13} /(\mathrm{AlN})_{13}$ junction forming a single quantum well. $(x z)$-plane averaged electronic potential and its average $\bar{V}(y)$, and conduction and valence band edges in the direct space relative to this potential are indicated by red lines. The band edges obtained from the local densities of states at Ga-N and Al-N bonds at different locations of the unit cell are also shown by dots. (c) Atomic structure of a regular patterned GaN/AlN core/shell array with the energy bands comprising flat confined states indicated by arrows. Reproduced with permission from Onen et al., Phys. Rev. B 95, 155435 (2017). Copyright 2017 American Physical Society.

lattice matched, can one construct new lateral and vertical composite structures and junctions with novel properties? Important conclusions drawn from several previous studies can be summarized as: (1) Free-standing, 2D SL GaN can form hexagonal and square-octagon structures. They have fundamental indirect band gaps, which are larger than the direct fundamental band gap of the parent 3D wz-crystals. (2) Similarly, free-standing 2D SL AlN can form hexagonal, tetragonal and square-octagon structures. 2D SL structures of AlN have indirect band gaps, which in contrast to SL GaN, are smaller than the direct band gap of 3D wz-AlN. (3) Fundamental band gaps of SL GaN and AlN decrease with increasing biaxial, in-plane strain. (4) SL GaN and AlN structures have cohesive energies smaller than that of 3D wz-GaN and wz-AlN, respectively. For h-GaN and hAlN, high temperature $a b$ initio MD simulations showed that the dynamical stability deduced by ab initio phonon calculations does not correspond to a shallow minimum, but the structures resist to thermal excitations by remaining stable at high temperatures. (5) The TDOS of 2D SL GaN and AlN are rather different from those of the 3D GaN and AlN crystals. Effects of dimensionality are reflected on the optical properties leading to important differences in the optical spectra between 2D and 3D structures. In particular, the absorption spectrum of $2 \mathrm{D} \mathrm{h}-\mathrm{GaN}$ is blueshifted and displays dramatic differences at higher photon energies. The amplitude and onset energy of the absorption spectrum, as well as the absorbance can be controlled by the number of layers. In general, prominent optical absorption kicks-off in the visible range for $\mathrm{h}-\mathrm{GaN}$ and occurs within the UVrange for both $\mathrm{h}-\mathrm{GaN}$ and $\mathrm{h}-\mathrm{AlN}$, promising for optoelectronic applications aimed for the visible-ultraviolet spectral regions. Also, the absorption spectrum is observed to globally redshift under the applied strain. (6) The planar geometry of SL GaN and AlN is maintained in their BL, which are constructed through $\mathrm{AA}^{\prime}$ stacking sequence without forming perpendicular cation-anion bonds between the layers. The indirect band gaps of $\mathrm{h}-\mathrm{GaN}$ and $\mathrm{h}-\mathrm{AlN}$ decrease and are converted into direct band gaps with increasing number of layers. Stable, 3D layered vdW structure with $\mathrm{AA}^{\prime} \mathrm{AA}^{\prime} \mathrm{A}$.. stacking sequence can be constructed from SL h-GaN and h-AlN. In a different study by Kolobov et al. ${ }^{28}$ however, it was predicted that the planar form of few-layer h-GaN layers is not the most favorable phase, yet it reconstructs into the haeckelite structure with covalent interlayer bonds. Observations as a result of the complementary efforts in this review article have been that $\mathrm{BL}$ GaN layers conformed with $\mathrm{AA}^{\prime}$ stacking do not transform into a haeckelite structure by themselves, whereas, once a $\mathrm{BL}$ or $3 \mathrm{D}$ periodic structure of $\mathrm{h}-\mathrm{GaN}$ is given an initial structure with interlayer haeckelite bonds, this is preserved with slightly lower interlayer interaction energies than the planar counterparts. (7) Since 3D layered GaN and AlN do not exist in nature, SL GaN and AlN should be grown on substrates. Physical properties of SL h-GaN and h-AlN predicted for their free-standing forms are not affected if they are grown on suitable substrates such as $\mathrm{Al}(111)$ and nearly lattice matched blue phosphorene. (8) Since SL GaN and AlN structures that are nearly lattice matched share the same anion, lateral and vertical composite structures of GaN/AlN can form and allow a diversity of novel devices, such as multiple and single quantum wells, and patterned core/shell structures.

The theoretical and experimental research motivated with the recent synthesis of very thin $2 \mathrm{D}$ AlN and GaN pose many interesting aspects, which should be addressed and clarified. Despite the fact that SL GaN and AlN and their multilayers display so many interesting physical and 
chemical properties, the diversity of these properties can be multiplied when these structures are functionalized by the adsorption of adatoms in specific decorations. Depending on the type of the adsorbed adatoms, the electronic structure as well as the optical properties of these SL or ML can be modified. Specific adatoms can attribute permanent dipole moments. ${ }^{145}$ Adsorption of metal adatoms according to a given pattern can make metallic interconnects on SL GaN and AlN. Through dilute chemisorption or substitution of adatoms, localized acceptor and donor states in the band gap can occur, ${ }^{118}$ whereby excess carriers can be attained in these 2D structures; the measurements on the mobilities of excess carriers appear to be important for further research related with 2D GaN and AlN optoelectronics. Not only adsorbates but also the creation of point defects like a vacancy is important, since, for instance, SL GaN or AlN structures acquire permanent magnetic moment through a vacancy of a $\mathrm{Ga}(\mathrm{Al})-\mathrm{N}$ pair.

While the works synthesizing ultrathin $2 \mathrm{D} \mathrm{GaN}$ and AlN have been seminal for these 2D structures, ${ }^{47,49}$ further theoretical and experimental works are needed for a better understanding of the growth processes and the structure of the grown layer. In particular, $\mathrm{Ga}_{2} \mathrm{~N}_{3}$ layer grown on $\mathrm{SiC}$ surface seems to be spin-polarized and have finite density of states at the Fermi level. Additionally, while SL h-GaN is planar, the overlayer grown between hydrogenated graphene and $\mathrm{SiC}$ surfaces are specified as buckled. We believe that the study of the growth of SL and few-layer h-GaN and hAlN on diverse substrates will shed light on the growth processes and substrate-overlayer interactions. Whether the growth of few-layer h-GaN and h-AlN constituted from planar single layers can be attained, or else vertical bonds between the cation and anion can form is a critical issue, which is essential for the fabrication of layered, synthetic GaN and AlN. In this respect, the growth of ML 2D h-GaN and h-AlN under perpendicular pressure can clarify whether perpendicular bonds between the layers can be constructed. Further work on growth is needed also in order to deduce substrates with minimum substrate-overlayer interactions. Growth on a substrate is also significant in order to develop techniques for printing circuits or specific patterns within or on $\mathrm{GaN}$ and AlN overlayers.

There exist further critical and fundamental issues, which ought to be clarified by further experimental and theoretical studies. One example is why the fundamental band gap of SL-AlN increases with the increasing number of layers in contrast to the trend in $\mathrm{h}-\mathrm{GaN}$.

Since SL h-AlN, h-GaN, and h-SiC are almost lattice matched semiconductors, they allow the commensurate growth of in-plane and vertical composite structures. These SL structures can also make junctions with metallic graphite and specific SL magnetic/nonmagnetic transition metal dichalcogenides. This situation opens a wide range of field of composite structures, novel synthetic materials with a diversity of the physical properties. One can achieve the formation of Schottky barriers, rectifiers, multiple and single quantum wells, resonant tunneling devices, patterned core/ shell structures using planar and vertical structures of these nearly lattice matched 2D materials. Not only semiconductor/ semiconductor junctions but also the metal/semiconductor/ metal junctions can operate as nanocapacitors. Moreover, a spin-valve effect can be attained from the metal/magneticmetal/metal junctions.

In summary, SL GaN and AlN, their multilayers and composites are promising materials, which deserve further extensive research for the development of novel devices.

\section{ACKNOWLEDGMENTS}

The computational resources are provided by TUBITAK ULAKBIM, High Performance and Grid Computing Center (TR-Grid e-Infrastructure), and the National Center for High Performance Computing of Turkey (UHeM) under Grant No. 5003622015. This work was supported by the Scientific and Technological Research Council of Turkey (TUBITAK) under Project No 115F088. E.D. acknowledges the financial support from the Turkish Academy of Sciences within Outstanding Young Scientists Award Program (TUBAGEBIP). S.C. acknowledges the financial support from the Turkish Academy of Sciences (TUBA). S. Cahangirov was supported by the BAGEP Award of the Science Academy.

${ }^{1}$ J. Bardeen and W. H. Brattain, Phys. Rev. 74, 230 (1948).

${ }^{2}$ W. Shockley, Bell Labs Tech. J. 28(3), 344-366 (1949).

${ }^{3}$ S. Nakamura, M. Takashi, and S. Masayuki, Appl. Phys. Lett. 64, 1687 (1994).

${ }^{4}$ S. Nakamura, S. Pearton, and G. Fasol, The Blue Laser Diode, 2nd ed. (Springer, New York, 2000), pp. 230-235.

${ }^{5}$ S. Nakamura, M. Senoh, S-i. Nagahama, N. Iwasa, T. Matsushita, and T. Mukai, Appl. Phys. Lett. 76, 22 (2000).

${ }^{6} \mathrm{H}$. Morkoc, Handbook of Nitride Semiconductors and Devices (WILEYVCH Verlag, Weinheim, 2008), Vol. 1.

${ }^{7}$ O. Ambacher, J. Phys. D: Appl. Phys. 31, 2653 (1998).

${ }^{8}$ S. Mokkapati and C. Jagadish, "III-V compound SC for optoelectronic devices," Mater. Today 12(4), 22-32 (2009).

${ }^{9}$ S. Nakamura, Rev. Mod. Phys. 87(4), 1139 (2015).

${ }^{10}$ K. S. Novoselov, A. K. Geim, S. V. Morozov, D. Jiang, Y. Zhang, S. V. Dubonos, I. V. Grigorieva, and A. A. Firsov, Science 306, 666 (2004).

${ }^{11}$ K. Watanabe, T. Taniguchi, and H. Kanda, Nat. Mater. 3, 404 (2004).

${ }^{12}$ K. Kim, A. Hsu, X. Jia, S. M. Kim, Y. Shi, M. Hofmann, D. Nezich, J. F. Rodriguez-Nieva, M. Dresselhaus, T. Palacios, and J. Kong, Nano Lett. 12, 161 (2012).

${ }^{13}$ Y. Gao, W. Ren, T. Ma, Z. Liu, Y. Zhang, W. B. Liu, L. P. Ma, X. Ma, and H. M. Cheng, ACS Nano 7, 5199 (2013).

${ }^{14}$ A. Splendiani, L. Sun, Y. Zhang, T. Li, J. Kim, C.-Y. Chim, G. Galli, and F. Wang, Nano Lett. 10, 1271 (2010).

${ }^{15}$ K. F. Mak, C. Lee, J. Hone, J. Shan, and T. F. Heinz, Phys. Rev. Lett. 105, 136805 (2010).

${ }^{16}$ G. Eda, H. Yamaguchi, D. Voiry, T. Fujita, M. Chen, and M. Chhowalla, Nano Lett. 11, 5111 (2011).

${ }^{17}$ E. Durgun, S. Tongay, and S. Ciraci, Phys. Rev. B 72, 075420 (2005).

${ }^{18}$ S. Cahangirov, M. Topsakal, E. Akturk, H. Sahin, and S. Ciraci, Phys. Rev. Lett. 102, 236804 (2009).

${ }^{19}$ S. Cahangirov, M. Topsakal, and S. Ciraci, Phys. Rev. B 81(19), 195120 (2010).

${ }^{20}$ H. Sahin, S. Cahangirov, M. Topsakal, E. Bekaroglu, E. Akturk, R. T. Senger, and S. Ciraci, Phys. Rev. B 80, 155453 (2009).

${ }^{21}$ C. L. Freeman, F. Claeyssens, N. L. Allan, and J. H. Harding, Phys. Rev. Lett. 96, 066102 (2006).

${ }^{22}$ Y. Wang and S. Shi, Solid State Commun. 150, 1473 (2010).

${ }^{23}$ Y. Ma et al., Appl. Surf. Sci. 257, 7845 (2011).

${ }^{24}$ A. K. Singh and R. G. Hennig, Appl. Phys. Lett. 105, 051604 (2014).

${ }^{25}$ A. K. Singh, H. L. Zhuang, and R. G. Hennig, Phys. Rev. B 89, 245431 (2014).

${ }^{26}$ Q. Chen, H. Hu, X. Chen, and J. Wang, Appl. Phys. Lett. 98, 053102 (2011).

${ }^{27}$ Y. Gao, T. Yayama, and S. Okada, Appl. Phys. Express 9, 095201 (2016). 
${ }^{28}$ A. V. Kolobov, P. Fons, J. Tominaga, B. Hyot, and B. Andre, Nano Lett. 16, 4849 (2016).

${ }^{29}$ D. Wu, M. G. Lagally, and F. Liu, Phys. Rev. Lett. 107, 236101 (2011).

${ }^{30}$ H. L. Zhuang, A. K. Singh, and R. G. Hennig, Phys. Rev. B 87, 165415 (2013).

${ }^{31}$ D. V. Fakhrabad, N. Shahtahmasebi, and M. Ashhadi, Superlattices Microstruct. 79, 38 (2015)

${ }^{32}$ C. Bacaksiz, H. Sahin, H. D. Ozaydin, S. Horzum, R. T. Senger, and F. M. Peeters, Phys. Rev. B 91, 085430 (2015).

${ }^{33}$ D. Kecik, C. Bacaksiz, R. T. Senger, and E. Durgun, Phys. Rev. B 92, 165408 (2015)

${ }^{34}$ A. Onen, D. Kecik, E. Durgun, and S. Ciraci, Phys. Rev. B 93, 085431 (2016)

${ }^{35}$ A. Onen, D. Kecik, E. Durgun, and S. Ciraci, Phys. Rev. B 95, 155435 (2017).

${ }^{36}$ A. Onen, D. Kecik, E. Durgun, and S. Ciraci, J. Phys. Chem. C 121, 27098 (2017).

${ }^{37}$ N. Sanders, D. Bayerl, G. Shi, K. A. Mengle, and E. Kioupakis, Nano Lett. 17, 7345 (2017).

${ }^{38}$ Z. Liliental-Weber, Y. Chen, S. Ruvimov, and J. Washburn, Phys. Rev. Lett. 79, 2835 (1997).

${ }^{39}$ J. Goldberger, R. He, Y. Zhang, S. Lee, H. Yan, H. Choi, and P. Yang, Nature 422, 599 (2003).

${ }^{40}$ V. N. Tondare, C. Balasubramanian, S. Shende, D. S. Joag, V. P. Godbale, and S. V. Bhoraskar, Appl. Phys. Lett. 80, 4813 (2002).

${ }^{41}$ Y. Mei, D. J. Thurmer, C. Deneke, S. Kiravittaya, Y.-F. Chen, A. Dadgar et.al., ACS Nano 3, 1663 (2009).

${ }^{42}$ Z. J. Reitmeier, S. Einfeldt, R. F. Davis, X. Zhang, X. Fang, and S. Mahajan, Acta Mater. 57, 4001 (2009).

${ }^{43}$ V. Kladko, A. Kuchuk, P. Lytvyn, O. Yefanov, N. Safriuk, A. Belyaev, Y. I. Mazur, E. A. Decuir, Jr., M. E. Ware, and G. J. Salamo, Nanoscale Res. Lett. 7, 289 (2012).

${ }^{44}$ T. Araki, S. Uchimura, J. Sakaguchi, Y. Nanishi, T. Fujishima, A. Hsu, K. K. Kim, T. Palacios, A. Pesquera, A. Centeno, and A. Zurutuza, Appl. Phys. Express 7, 071001 (2014)

${ }^{45}$ P. Motamedi, N. Dalili, and K. Cadien, J. Mater. Chem. C 3, 7428 (2015).

${ }^{46}$ T. H. Seo, A. H. Park, S. Park, Y. H. Kim, G. H. Lee, M. J. Kim, M. S. Jeong, Y. H. Lee, Y. B. Hahn, and E. K. Suh, Sci. Rep. 5, 7747 (2015).

${ }^{47}$ P. Tsipas, S. Kassavetis, D. Tsoutsou, E. Xenogiannopoulou, E. Golias, S A. Giamini, C. Grazianetti, D. Chiappe, A. Molle, M. Fanciulli, and A. Dimoulas, Appl. Phys. Lett. 103, 251605 (2013).

${ }^{48}$ V. Mansurov, T. Malin, Y. Galitsyn, and K. Zhuravlev, J. Cryst. Growth 428, 93 (2015)

${ }^{49}$ Z. Y. Al Balushi, K. Wang, R. K. Ghosh, R. A. Vila, S. M. Eichfeld, J. D. Caldwell, X. Qin, Y.-C. Lin, P. A. DeSario, G. Stone, S. Subramanian, D. F. Paul, R. M. Wallace, S. Datta, J. M. Redwing, and J. A. Robinson, Nature Mater. 15, 1166 (2016).

${ }^{50}$ M. Magnuson, M. Mattesini, C. Hoglund, J. Birch, and L. Hultman, Phys. Rev. B 81, 085125 (2010).

${ }^{51}$ H. Schulz and K. H. Thiemann, Solid State Commun. 23, 815 (1977).

${ }^{52}$ I. Vurgaftman and J. R. Meyer, J. Appl. Phys. 94, 3675 (2003).

${ }^{53}$ H. Xia, Q. Xia, and A. L. Ruoff, Phys. Rev. B 47, 12925 (1993).

${ }^{54}$ M. Ueno, M. Yoshida, A. Onodera, O. Shimomura, and K. Takemura, Phys. Rev. B 49, 14 (1994).

${ }^{55}$ P. Perlin, C. Jauberthie-Carillon, J. P. Itie, A. San Miguel, I. Grzegory, and A. Polian, Phys. Rev. B 45, 83 (1992).

${ }^{56}$ C. Bungaro, K. Rapcewicz, and J. Bernholc, Phys. Rev. B 61, 6720 (2000)

${ }^{57}$ A. Wright and J. Nelson, Phys. Rev. B 50, 2159 (1994); 51, 7866 (1995).

${ }^{58}$ A. Polian, M. Grimsditch, and I. Grzegory, J. Appl. Phys. 79, 3343 (1996).

${ }^{59}$ F. A. Ponce and D. P. Bour, Nature 386, 351 (1997).

${ }^{60}$ L. Hultman, S. Benhenda, G. Radnoczi, J.-E. Sundgren, J. E. Greene, and I. Petrov, Thin Solid Films 215, 152 (1992).

${ }^{61}$ M. Ueno, A. Onodera, O. Shimomura, and K. Takemura, Phys. Rev. B 45, 10123 (1992).

${ }^{62}$ Q. Xia, H. Xia, and A. L. Ruoff, J. Appl. Phys. 73, 8198 (1993).

${ }^{63}$ A. V. Virkar, T. B. Jackson, and R. A. Cutler, J. Am. Ceram. Soc. 72 2031 (1989).

${ }^{64}$ A. W. Weimer, G. A. Cochran, G. A. Eisman, J. P. Henley, B. D. Hook, L. K. Mills, T. A. Guiton, A. K. Knudsen, N. R. Nicholas, J. E. Volmering, and W. G. Moor, J. Am. Ceram. Soc. 77, 3 (1994).

${ }^{65}$ G. A. Slack, R. A. Tanzilli, and R. O. Pohl, J. Phys. Chem. Solids 48, 141 (1987).
${ }^{66}$ I. H. Nwigboji, J. I. Ejembi, Y. Malozovsky, B. Khamala, L. Franklin, G. Zhao, C. E. Ekuma, and D. Bagayoko, Mater. Chem. Phys. 157, 80-86 (2015).

${ }^{67}$ F. Litimein, B. Bouhafs, Z. Dridi, and P. Ruterana, New J. Phys. 4, 64 (2002).

${ }^{68}$ P. Vashishta, R. K. Kalia, A. Nakano, and J. P. Rino, J. Appl. Phys. 109, 033514 (2011).

${ }^{69}$ I. Akasaki and H. Amano, Jpn. J. Appl. Phys., Part 1 36, 5393 (1997).

${ }^{70}$ J. Han, M. H. Crawford, R. J. Shul, J. J. Figiel, M. Banas, L. Zhang, Y. K. Song, H. Zhou, and A. V. Nurmikko, Appl. Phys. Lett. 73, 1688 (1998).

${ }^{71}$ T. Nishida and N. Kobayashi, Phys. Status Solidi A 176, 45 (1999).

${ }^{72}$ V. Adivarahan, W. H. Sun, A. Chitnis, M. Shatalov, S. Wu, H. P. Maruska, and M. A. Khan, Appl. Phys. Lett. 85, 2175 (2004).

${ }^{73}$ H. Hirayama, J. Appl. Phys. 97, 091101 (2005).

${ }^{74}$ A. Khan, K. Balakrishnan, and T. Katona, Nature Photon. 2, 77 (2008).

${ }^{75}$ G. Ramirez-Flores, H. Navarro-Contreras, A. Lastras-Martinez, R. C. Powell, and J. E. Greene, Phys. Rev. B 50, 8433 (1994).

${ }^{76}$ M. E. Sherwin and T. J. Drummond, J. Appl. Phys. 69, 8423 (1991).

${ }^{77}$ R. C. Weast, CRC Handbook of Chemistry and Physics (CRC Press, Boca Raton, Florida, 1988), Vol. 68.

${ }^{78}$ E. Sanville, S. D. Kenny, R. Smith, and G. Henkelman, J. Comput. Chem. 28, 899 (2007).

${ }^{79}$ Q. Yan, P. Rinke, A. Janotti, M. Scheffler, and C. G. Van de Walle, Phys. Rev. B 90, 125118 (2014).

${ }^{80}$ L. C. de Carvalho, A. Schleife, and F. Bechstedt, Phys. Rev. B 84, 195105 (2011).

${ }^{81}$ K. Karch, J. M. Wagner, and F. Bechstedt, Phys. Rev. B 57, 7043 (1998).

${ }^{82}$ F. Bernardini, V. Fiorentini, and D. Vanderbilt, Phys. Rev. B 56(16), R10024 (1997)

${ }^{83}$ F. Bechstedt, U. Grossner, and J. Furthmüller, Phys. Rev. B 62, 8003 (2000).

${ }^{84}$ M. A. Caro, S. Schulz, and E. P. O'Reilly, Phys. Rev. B 88, 214103 (2013).

${ }^{85}$ Z. Dridi, B. Bouhafs, and P. Ruterana, Semicond. Sci. Technol. 18, 850 (2003).

${ }^{86}$ J. Heyd, J. E. Peralta, G. E. Scuseria, and R. L. Martin, J. Chem. Phys. 123, 174101 (2005).

${ }^{87}$ A. Punya and W. R. L. Lambrecht, Phys. Rev. B 85, 195147 (2012).

${ }^{88}$ P. Rinke, M. Winkelnkemper, A. Qteish, D. Bimberg, J. Neugebauer, and M. Scheffler, Phys. Rev. B 77, 075202 (2008).

${ }^{89}$ Y. Du, B. Chang, X. Fu, X. Wang, and M. Wang, Optik 123, 2208 (2012).

${ }^{90}$ X.-Z. Li, R. Gomez-Abal, H. Jiang, C. Ambrosch-Draxl, and M. Scheffler, New J. Phys. 14, 023006 (2012).

${ }^{91}$ M. Rohlfing, P. Kruger, and J. Pollmann, Phys. Rev. B 57, 6485 (1998).

${ }^{92}$ P. Rinke, A. Qteish, J. Neugebauer, C. Freysoldt, and M. Scheffler, New J. Phys. 7, 126 (2005).

${ }^{93}$ F. Bechstedt, K. Seino, P. Hahn, and W. Schmidt, Phys. Rev. B 72(24), 245114 (2005)

${ }^{94}$ Y. C. Cheng, X. L. Wu, J. Zhu, L. L. Xu, S. H. Li, and P. K. Chu, J. Appl. Phys. 103, 073707 (2008).

${ }^{95}$ W. Yong-Liang, A. Qiong, C. Xiang-Rong, and C. Ling-Cang, Chin. Phys. 16(12), 3783 (2007).

${ }^{96}$ R. Mohammad and Ş. Katircioglu, Turk. J. Phys. 40, 219-230 (2016).

${ }^{97}$ R. Thokala and J. Chaudhuri, Thin Solid Films 266(2), 189-191 (1995).

${ }^{98}$ A. Bakhtatou and A. Meddour, Phys. Status Solidi B 253, 442 (2016).

${ }^{99}$ N. E. Christensen and I. Gorczyca, Phys. Rev. B 50, 4397 (1994).

${ }^{100}$ U. P. Verma and P. S. Bisht, Solid State Sci. 12, 665 (2010).

${ }^{101}$ J. Serrano, A. Rubio, E. Hernandez, and A. Munoz, Phys. Rev. B 62, $16612(2000)$

${ }^{102}$ S. Bagci, S. Duman, H. M. Tütüncü, and G. P. Srivastava, Diamond Relat. Mater. 18, 1057 (2009).

${ }^{103}$ I. Petrov, E. Mojab, R. C. Powell, J. E. Greene, L. Hultman, and J. E. Sundgren, Appl. Phys. Lett. 60(20), 2491 (1992).

${ }^{104}$ M. Röppischer, R. Goldhahn, G. Rossbach, P. Schley, C. Cobet, N. Esser, T. Schupp, K. Lischka, and D. J. As, J. Appl. Phys. 106, 076104 (2009).

${ }^{105}$ Z. Wei, C. Xiang-Rong, C. Ling-Cang, and G. Qing-Quan, Commun. Theor. Phys. 50, 990 (2008).

${ }^{106}$ R. Pandey, A. Sutjianto, M. Seel, and J. E. Jaffe, J. Mater. Res. 8, 1922 (1993).

${ }^{107}$ D. Xu, H. He, R. Pandey, and S. P. Karna, J. Phys.: Condens. Matter 25, 345302 (2013).

${ }^{108}$ D. C. Camacho-Mojica and F. Lopez-Urias, Sci. Rep. 5, 17902 (2015). 
${ }^{109}$ E. Gürbüz, S. Cahangirov, E. Durgun, and S. Ciraci, Phys. Rev. B 96, 205427 (2017).

${ }^{110}$ H. Zhang, F.-S. Meng, and Y.-B. Wu, Solid State Commun. 250, 18 (2017).

${ }^{111}$ Q. Fang, Y. Huang, Y. Miao, K. Xu, Y. Li, and F. Ma, J. Phys. Chem. C 121, 6605 (2017).

${ }^{112}$ Q. Peng, C. Liang, W. Ji, and S. De, Appl. Phys. A 113, 483 (2013).

${ }^{113}$ C. Attaccalite, A. Nguer, E. Cannuccia, and M. Gruning, Phys. Chem. Chem. Phys. 17, 9533 (2015).

${ }^{114}$ M. S. Prete, A. M. Conte, P. Gori, F. Bechstedt, and O. Pulci, Appl. Phys. Lett. 110, 012103 (2017).

${ }^{115}$ R. B. dos Santos, F. de Brito Mota, R. Rivelino, A. KakanakovaGeorgieva, and G. K. Gueorguiev, Nanotechnology 27, 145601 (2016).

${ }^{116}$ C.-J. Tong, H. Zhang, Y.-N. Zhang, H. Liu, and L.-M. Liu, J. Mater. Chem. A 2, 17971 (2014)

${ }^{117}$ P. A. Brown and K. L. Shuford, Nanoscale 8, 19287 (2016).

${ }^{118}$ F. Ersan, A. Akcay, G. Gokoglu, and E. Akturk, Chem. Phys. 455, 73-80 (2015).

${ }^{119}$ A. V. Kolobov, P. Fons, Y. Saito, J. Tominaga, B. Hyot, and B. Andre, Phys. Rev. Mater. 1, 024003 (2017).

${ }^{120}$ H. Shi, H. Pan, Y.-W. Zhang, and B. I. Yakobson, Phys. Rev. B 87, 155304 (2013).

${ }^{121}$ C. Kittel, Introduction to Solid State Physics, 7th ed. (John Wiley \& Sons Inc., New York, 1986).

${ }^{122}$ S. Cahangirov, V. O. Ozcelik, L. Xian, J. Avila, S. Cho, M. C. Asensio, S. Ciraci, and A. Rubio, Phys. Rev. B 90, 035448 (2014).

${ }^{123}$ S. Cahangirov, V. O. Ozcelik, A. Rubio, and S. Ciraci, Phys. Rev. B 90, 085426 (2014)

${ }^{124}$ A. K. Geim and I. V. Grigorieva, Nature 499, 419-425 (2013).

${ }^{125}$ V. Tran, R. Soklaski, Y. Liang, and L. Yang, Phys. Rev. B 89, 235319 (2014).

${ }^{126}$ D. Y. Qiu, F. H. Jornada, and S. G. Louie, Phys. Rev. Lett. 111, 216805 (2013).
${ }^{127}$ L. Wirtz, A. Marini, and A. Rubio, Phys. Rev. Lett. 96, 126104 (2006).

${ }^{128}$ J. Li, K. B. Nam, M. L. Nakarmi, J. Y. Lin, H. X. Jiang, P. Carrier, and S.-H. Wei, Appl. Phys. Lett. 83, 5163 (2003).

${ }^{129}$ E. Silveira, J. A. Freitas, Jr., M. Kneissl, D. W. Treat, N. M. Johnson, G. A. Slack, and L. J. Schowalter, Appl. Phys. Lett 84(18), 3501 (2004).

${ }^{130}$ M. Feneberg, M. F. Romero, M. Roppischer, C. Cobet, N. Esser, B. Neuschl, K. Thonke, M. Bickermann, and R. Goldhahn, Phys. Rev. B 87, 235209 (2013).

${ }^{131}$ A. Riefer, F. Fuchs, C. Rodl, A. Schleife, and F. Bechstedt, Phys. Rev. B 84, 075218 (2011).

${ }^{132}$ S. Ismail-Beigi, Phys. Rev. B 77, 035306 (2008).

${ }^{133}$ K. S. Thygesen, 2D Mater. 4, 022004 (2017).

${ }^{134}$ X. Y. Cui, B. Delley, and C. Stampfl, J. Appl. Phys. 108, 103701 (2010).

${ }^{135}$ N. Nepal, V. D. Wheeler, T. J. Anderson, F. J. Kub, M. A. Mastro, R. L. Myers-ward, S. B. Qadri et al., Appl. Phys. Express 6, 061003 (2013).

${ }^{136}$ D. Bayerl, S. Islam, C. M. Jones, V. Protasenko, D. Jena, and E. Kioupakis, Appl. Phys. Lett. 109, 241102 (2016)

${ }^{137}$ H. Sevincli, M. Topsakal, and S. Ciraci, Phys. Rev. B 78, 245402 (2008).

${ }^{138}$ M. Topsakal, H. Sevincli, and S. Ciraci, Appl. Phys. Lett. 92, 173118 (2008).

${ }^{139}$ V. O. Ozcelik, E. Durgun, and S. Ciraci, J. Phys. Chem. C 119, 13248 (2015).

${ }^{140}$ V. O. Ozcelik and S. Ciraci, Phys. Rev. B 91, 195445 (2015).

${ }^{141}$ M. Aras, C. Kilic, and S. Ciraci, Phys. Rev. B 95, 075434 (2017).

${ }^{142}$ G.-X. Chen, X.-G. Li, Y.-P. Wang, J. N. Fry, and H.-P. Cheng, Phys. Rev. B 95, 045302 (2017).

${ }^{143}$ P. Strak, P. Kempisty, M. Ptasinska, and S. Krukowsk, J. Appl. Phys. 113, 193706 (2013).

${ }^{144}$ Q. Chen, R. Song, C. Chen, and X. Chen, Solid State Commun. 172, 24 (2013).

${ }^{145}$ Y. Mu, J. Phys. Chem. C 119, 20911-20916 (2015). 